\title{
THE CONLEY INDEX FOR FLOWS PRESERVING GENERALIZED SYMMETRIES
}

\author{
ARTUR M. PRUSZKO \\ Institute of Mathematics, Polish Academy of Sciences \\ Abrahama 18, 81-825 Sopot, Poland \\ E-mail: A.Pruszko@impan.gda.pl
}

\begin{abstract}
Topological spaces with generalized symmetries are defined and extensions of the Conley index of a compact isolated invariant set of the flow preserving the structures introduced are proposed. One of the two new indexes is constructed with no additional assumption on the examined set in terms of symmetry invariance.

1. Introduction. The Conley index theory is developed in many different directions to examine various aspects of the behaviour of flows. It is well known that the behaviour of flows which admit certain symmetries is strongly limited. The works of A. Floer $[\mathrm{F}]$, A. Floer \& E. Zehnder [FZ] and T. Bartsch [B] show how the ideas of Conley index can be used to investigate the flows which are equivariant with respect to a compact Lie group action. Nevertheless, there appear some "symmetries" which cannot be obtained as a compact Lie group action.

The main aim of this paper is to study whether it is possible to define a homotopy index for flows which can contain some information about less regular symmetries than those indicated by a compact Lie group action. The positive answer is obtained through the concept of topological spaces with generalized symmetries. The suggested invariant is the homotopy type of a suitable compact pointed space with generalized symmetries.

The paper is organized as follows. In section 2 we introduce the notion of a topological space with generalized symmetries (or symmetries for short) over some base $\mathcal{B}$, a map preserving the generalized symmetries and finally the homotopy type of spaces with generalized symmetries. We discuss the basic properties of these objects. In particular we describe the class of regular symmetries which can be treated similarly to the "symmetries" established by the action of a compact Lie group.
\end{abstract}

1991 Mathematics Subject Classification: Primary 34C35; Secondary 54H20.

Research supported by KBN grant 0008/P03/96/11.

The paper is in final form and no version of it will be published elsewhere. 
Section 3 describes some relations between extensively investigated classes of flows, i.e. flows equivariant with respect to a compact Lie group action, flows admitting a first integral, flows commuting with another given flow and the class of flows preserving generalized symmetries. We show the way generalized symmetries can be defined in these cases.

In section 4 there are some illustrations of the notion of homotopy type of spaces with generalized symmetries.

Section 5 attempts to define the Conley index of compact isolated invariant sets of a flow which preserves symmetries. In fact, we define the index $\Sigma^{\star} h(\cdot)$ which can be associated with any compact isolated invariant set of this flow and the index $\Sigma h(\cdot)$ which is defined for some $\Sigma$-admissible compact isolated invariant sets. Next, we discuss the properties of those indexes. Finally, we consider regular symmetries. In this case we can describe in detail the class of $\Sigma$-admissible compact isolated invariant sets.

The proofs presented are based on the ideas of C.C. Conley [C] and standard constructions used in the Conley index theory which are excellently described in works of D. Salamon [S], J. Robbin \& D. Salamon [RS].

The last section 6 contains examples which show how these new invariants can be used to answer natural questions about the flow behaviour.

It is worth emphasizing that the concept of Conley index with generalized symmetries can be extended to the case of the Conley index for discrete semidynamical systems in the setting due to A. Szymczak $[\mathrm{Sz}]$.

This paper is a part of the author's Ph.D. thesis written at the Institute of Mathematics of the Polish Academy of Science. I would like to express my special gratitude to Professor K. Gęba who introduced me to this area of research. I also thank Professor L. Górniewicz whose remarks helped me to simplify some of the ideas presented.

2. Generalized symmetries in topological spaces. This part is devoted to the description of generalized symmetries in topological spaces and their basic properties.

Let $(X, \Theta)$ denote a Hausdorff topological space endowed with a topology $\Theta$ and let $P(X)$ stand for the family of subsets of $X$. Furthermore, for any subset $A \subset X, \operatorname{cl}(A)$ and $\operatorname{int}(A)$ denote the closure and the interior of $A$ according to the topology $\Theta$.

A map between topological spaces will always mean a continuous map unless explicitly stated otherwise.

We now formulate the definition of generalized symmetries in relation to some given abstract base set $\mathcal{B}$.

Definition 2.1. The generalized symmetries in $X$ over the base set $\mathcal{B}$ is any family

$$
\Sigma \subset(P(X) \backslash\{\emptyset\}) \times \mathcal{B} .
$$

The system $(X ; \Sigma, \mathcal{B})$ or $(X, A ; \Sigma, \mathcal{B})$, where $A \subset X$, is called a space with generalized symmetries over $\mathcal{B}$.

In the sequel we use the name symmetries instead of generalized symmetries; this will not lead to any confusion as we only consider generalized symmetries. 
Now, consider a given space with symmetries $(X, \Sigma ; \mathcal{B})$. We denote elements of $\Sigma$ by $a, b, c, \ldots$ Moreover, for $a=(|a|,(a)) \in \Sigma$, we will say that the subset $|a| \subset X$ is the support of $a$ and $(a) \in \mathcal{B}$ is the index or type of $a$.

For any subset $A \subset X$, we define the symmetries $\Sigma_{A}$ in $A$ induced by the symmetries $\Sigma$ as follows:

$$
\Sigma_{A} \stackrel{\text { def }}{=}\{(A \cap|a|,(a)): a \in \Sigma \text { and }|a| \cap A \neq \emptyset\} .
$$

In this sense we will consider the space with symmetries $\left(A ; \Sigma_{A}, \mathcal{B}\right)$ as a subspace of $(X ; \Sigma, \mathcal{B})$.

Subsets $A, B \subset X$ are said to be $\Sigma$-disjoint if they are disjoint and $|a| \subset X \backslash A$ or $|a| \subset X \backslash B$ for any $a \in \Sigma$. This property implies that $\Sigma_{A \cup B}=\Sigma_{A} \cup \Sigma_{B}$. A subset $A \subset X$ is $\Sigma$-invariant if $A$ and $X \backslash A$ are $\Sigma$-disjoint. Let $P_{\Sigma}(X)$ stand for the family of $\Sigma$-invariant subsets of $X$.

Proposition 2.2. The family $P_{\Sigma}(X)$ forms an algebra of sets with the natural operations $\cup$ and $\cap$, i.e.

(1) $\emptyset \in P_{\Sigma}(X)$ and $X \in P_{\Sigma}(X)$,

(2) $X \backslash A \in P_{\Sigma}(X)$ for any $A \in P_{\Sigma}(X)$,

(3) $\bigcup \mathcal{A} \in P_{\Sigma}(X)$ and $\bigcap \mathcal{A} \in P_{\Sigma}(X)$ for any family $\mathcal{A} \subset P_{\Sigma}(X)$.

Thus we can formulate the following definition.

Definition 2.3. The family of sets

$$
\Theta_{\Sigma} \stackrel{\text { def }}{=} \Theta \cap P_{\Sigma}(X)
$$

is called the topology of $\Sigma$-invariant sets.

To complete the formal definitions we introduce the notion of sum and product of spaces with symmetries.

Definition 2.4. Let $\left(X, A ; \Sigma_{X}, \mathcal{B}_{X}\right)$ and $\left(Y, B ; \Sigma_{Y}, \mathcal{B}_{Y}\right)$ be spaces with symmetries. We define

- the sum

$$
\left(X, A ; \Sigma_{X}, \mathcal{B}_{X}\right) \cup\left(Y, B ; \Sigma_{Y}, \mathcal{B}_{Y}\right) \stackrel{\text { def }}{=}\left(X \sqcup Y, A \cup B ; \Sigma_{X} \cup \Sigma_{Y}, \mathcal{B}_{X} \cup \mathcal{B}_{Y}\right),
$$

if only the spaces $X$ and $Y$ are disjoint, where $\sqcup$ means topological sum,

- the product

$$
\left(X, A ; \Sigma_{X}, \mathcal{B}_{X}\right) \times\left(Y, B ; \Sigma_{Y}, \mathcal{B}_{Y}\right) \stackrel{\text { def }}{=}\left(X \times Y, X \times B \cup A \times Y ; \Sigma_{X \times Y}, \mathcal{B}_{X} \times \mathcal{B}_{Y}\right),
$$

where $\Sigma_{X \times Y}=\left\{(|a| \times|b|,((a),(b))): a \in \Sigma_{X}, b \in \Sigma_{Y}\right\}$.

Remark 2.5. If $A \in P_{\Sigma_{X}}(X)$ and $B \in P_{\Sigma_{Y}}(Y)$, then $A \times B \in P_{\Sigma_{X \times Y}}(X \times Y)$.

Proof. Indeed, let $c \in \Sigma_{X \times Y}$ and $|c| \cap A \times B \neq \emptyset$. Then

$$
c=(|a| \times|b|,((a),(b))),|a| \cap A \neq \emptyset \text { and }|b| \cap B \neq \emptyset
$$

for some $a \in \Sigma_{X}$ and $b \in \Sigma_{Y}$. Hence $|a| \subset A$ and $|b| \subset B$, as they are $\Sigma$-invariant subsets. Thus $|c| \subset A \times B$. 
2.1. Regular symmetries. Consider a space with symmetries $(X ; \Sigma, \mathcal{B})$. The symmetries $\Sigma$ define the relation $R_{\Sigma} \subset X \times X$ by

$$
R_{\Sigma}=\left\{(x, y) \in X \times X: \exists_{a \in \Sigma} x, y \in|a|\right\} .
$$

It is elementary to verify that this is an equivalence relation if, for instance, the symmetries $\Sigma$ satisfy the following conditions:

- $X=\bigcup_{a \in \Sigma}|a|$,

- for any $a, b \in \Sigma$ we have $|a|=|b|$ or $|a| \cap|b|=\emptyset$.

If $R_{\Sigma}$ is an equivalence relation then the quotient space $X / R_{\Sigma}$ is a topological space and the canonical projection $\pi: X \rightarrow X / R_{\Sigma}$ is a continuous map.

REMARK 2.6. If $R_{\Sigma}$ is an equivalence relation then subsets $A, B \subset X$ are $\Sigma$-disjoint if and only if $\pi(A) \cap \pi(B)=\emptyset$.

Proof. $(\Rightarrow)$ If $z \in \pi(A) \cap \pi(B)$, then there are $x \in A$ and $y \in B$ such that $z=$ $\pi(x)=\pi(y)$. Hence, there must exist $a \in \Sigma$ such that $x, y \in|a|$, so $|a| \cap A \neq \emptyset \neq|a| \cap B$, which contradicts our assumption.

$(\Leftarrow)$ Let $a \in \Sigma$ and $|a| \cap A \neq \emptyset$. Then

$$
|a| \subset \pi^{-1}(\pi(A)) \subset X \backslash \pi^{-1}(\pi(B)) \subset X \backslash B,
$$

as $\pi^{-1}(\pi(A)) \cap \pi^{-1}(\pi(B))=\emptyset$ and $B \subset \pi^{-1}(\pi(B))$.

COROLlary 2.7. If $R_{\Sigma}$ is an equivalence relation then

$$
A \in P_{\Sigma}(X) \text { if and only if } A=\pi^{-1}(\pi(A)) \text {. }
$$

Definition 2.8. The symmetries $\Sigma$ are regular if $R_{\Sigma}$ is an equivalence relation and the canonical projection $\pi: X \rightarrow X / R_{\Sigma}$ is a closed and open mapping.

We recall the following theorem.

THEOREM 2.9. If $X$ is a locally compact metric space and the symmetries $\Sigma$ are regular then the quotient space $X / R_{\Sigma}$ is a locally compact metric space.

Proof. It is known [E, theorem 4.4.18] that the quotient topology is metrizable as there is a closed and open surjection $\pi: X \rightarrow X / R_{\Sigma}$.

Moreover, the quotient space $X / R_{\Sigma}$ is locally compact, as the quotient topology is metrizable and $\pi: X \rightarrow X / R_{\Sigma}$ is an open surjection, see [E, theorem 3.3.15].

2.2. Maps preserving generalized symmetries. The additional structure of symmetries allows us to describe the class of maps which preserve the symmetries introduced.

Definition 2.10. Let $\left(X, A ; \Sigma_{X}, \mathcal{B}\right)$ and $\left(Y, B ; \Sigma_{Y}, \mathcal{B}\right)$ be spaces with symmetries. A continuous map $f:(X, A) \rightarrow(Y, B)$ is $\Sigma$-invariant (preserves the symmetries) if for any $a \in \Sigma_{X}$ there is $b \in \Sigma_{Y}$ satisfying

$$
f(|a|) \subset|b| \text { and }(a)=(b) .
$$

We write

$$
f:\left(X, A ; \Sigma_{X}, \mathcal{B}\right) \rightarrow\left(Y, B, \Sigma_{Y}, \mathcal{B}\right)
$$


A $\Sigma$-invariant map is called a $\Sigma$-homeomorphism if its inverse is also a $\Sigma$-invariant map.

Proposition 2.11. The basic properties of $\Sigma$-invariant maps are:

(1) the identity map id $:\left(X, A ; \Sigma_{X}, \mathcal{B}\right) \rightarrow\left(X, A ; \Sigma_{X}, \mathcal{B}\right)$ is $\Sigma$-invariant,

(2) for any $\Sigma$-invariant maps

$$
f:\left(X, A ; \Sigma_{X}, \mathcal{B}\right) \rightarrow\left(Y, B ; \Sigma_{Y}, \mathcal{B}\right) \text { and } g:\left(Y, B ; \Sigma_{Y}, \mathcal{B}\right) \rightarrow\left(Z, C ; \Sigma_{Z}, \mathcal{B}\right)
$$

the composition $g \circ f:\left(X, A ; \Sigma_{X}, \mathcal{B}\right) \rightarrow\left(Z, C ; \Sigma_{Z}, \mathcal{B}\right)$ is $\Sigma$-invariant,

(3) for any $\Sigma$-invariant map $f:\left(X, A ; \Sigma_{X}, \mathcal{B}\right) \rightarrow\left(Y, B, \Sigma_{Y}, \mathcal{B}\right)$ the restriction

$$
f_{\mid A}:\left(A ; \Sigma_{A}, \mathcal{B}\right) \rightarrow\left(B ; \Sigma_{B}, \mathcal{B}\right), f_{\mid A}(x) \stackrel{\text { def }}{=} f(x)
$$

is $\Sigma$-invariant with respect to the induced symmetries.

REMARK 2.12. Let $f:\left(X ; \Sigma_{X}, \mathcal{B}\right) \rightarrow\left(Y ; \Sigma_{Y}, \mathcal{B}\right)$ and $B \in P_{\Sigma_{Y}}(Y)$ be a $\Sigma_{Y}$-invariant subset of $Y$. Then the preimage $f^{-1}(B) \in P_{\Sigma_{X}}(X)$ is a $\Sigma_{X}$-invariant subset of $X$.

Proof. Let $a \in \Sigma_{X}$ be such that $|a| \cap f^{-1}(B) \neq \emptyset$. As $f$ preserves the symmetries, $f(|a|) \subset|b|$ for some $b \in \Sigma_{Y}$, so $|b| \cap B \neq \emptyset$. Hence $|b| \subset B$ as $B \in P_{\Sigma_{Y}}(Y)$, thus $|a| \subset f^{-1}(B)$.

2.3. Topological spaces with base point and generalized symmetries. We say that the symmetries $\Sigma_{X}$ in the space $X$ agree with the base point $\star \in X$ when $(\{\star\}, \tau) \in \Sigma_{X}$ for any $\tau \in \mathcal{B}$. In this case we write $\left(X, \star ; \Sigma_{X}, \mathcal{B}\right)$.

This condition implies that for any space $\left(Y ; \Sigma_{Y}, \mathcal{B}\right)$ the constant map

$$
f:\left(Y ; \Sigma_{Y}, \mathcal{B}\right) \rightarrow\left(X, \star ; \Sigma_{X}, \mathcal{B}\right), f(x) \stackrel{\text { def }}{=} \star
$$

is $\Sigma$-invariant.

Furthermore, we define the pointed symmetries

$$
\left(X, \star ; \Sigma_{X}, \mathcal{B}\right)^{\star} \stackrel{\text { def }}{=}\left(X, \star ; \Sigma_{X}^{\star}, \mathcal{B}\right)
$$

where $\Sigma_{X}^{\star}=\left\{(|a| \cup\{\star\},(a)): a \in \Sigma_{X}\right\}$.

RemaRK 2.13. If the map $f:\left(X, \star ; \Sigma_{X}, \mathcal{B}\right) \rightarrow\left(Y, \star ; \Sigma_{Y}, \mathcal{B}\right)$ preserves the symmetries, then $f:\left(X, \star ; \Sigma_{X}^{\star}, \mathcal{B}\right) \rightarrow\left(Y, \star ; \Sigma_{Y}^{\star}, \mathcal{B}\right)$ preserves the pointed symmetries.

Proof. Let $a \in \Sigma_{X}^{\star}$. Then $a=(|b| \cup\{\star\},(b))$ for some $b \in \Sigma_{X}$. By our assumption, there is $c \in \Sigma_{Y}$ such that $(c)=(a)$ and $f(|b|) \subset|c|$, hence $f(|a|) \subset|c| \cup\{\star\}$.

2.3.1. Quotient space of a pair of sets. An important example of a topological space with base point is a space generated by a pair of sets.

For any pair $(X, A)$ of sets, $A \subset X$, there is the quotient space defined by

$$
X / A \stackrel{\text { def }}{=} X \backslash A \cup\{A\}
$$

and the canonical projection

$$
p: X \rightarrow X / A, p(x)= \begin{cases}x & \text { if } x \notin A \\ A & \text { if } x \in A .\end{cases}
$$

A set $V \subset X / A$ is open if and only if its preimage $p^{-1}(V)$ is an open subset of $X$. 
The point $\star=[A] \stackrel{\text { def }}{=} A$ is the base point of $X / A$ corresponding to the subset $A$ and we write

$$
Q(X, A) \stackrel{\text { def }}{=}(X / A, A) .
$$

It is obvious that if $A \subset X$ is a compact subset of a Hausdorff space $X$ then $X / A$ is a Hausdorff topological space. It is sufficient for further considerations to assume that $A$ is compact.

This construction leads us to the notion of the quotient spaces with symmetries induced by pairs of sets.

Definition 2.14. Let $A \subset X$ be a compact subset of $X$. We call

$$
Q\left(X, A ; \Sigma_{X}, \mathcal{B}\right) \stackrel{\text { def }}{=}\left(X / A, \star ; \Sigma_{X / A}, \mathcal{B}\right)
$$

where

$$
\Sigma_{X / A} \stackrel{\text { def }}{=}\left\{(p(|a|),(a)): a \in \Sigma_{X}\right\} \cup\{\{\star\}\} \times \mathcal{B},
$$

the quotient space with symmetries induced by the pair $(X, A)$.

Moreover, we call

$$
Q^{\star}\left(X, A ; \Sigma_{X}, \mathcal{B}\right) \stackrel{\text { def }}{=} Q\left(X, A ; \Sigma_{X}, \mathcal{B}\right)^{\star}
$$

the weak quotient space with symmetries induced by the pair $(X, A)$.

2.3.2. Sum and product of spaces with base points and generalized symmetries. Now, we define the sum and product of spaces with base points in the context of spaces with symmetries.

Definition 2.15. Let $\left(X, \star, \Sigma_{X}, \mathcal{B}_{1}\right)$ and $\left(Y, \star ; \Sigma_{Y}, \mathcal{B}_{2}\right)$ be spaces with symmetries.

- If $X$ and $Y$ are disjoint then we define the sum

$$
\left(X, \star ; \Sigma_{X}, \mathcal{B}_{1}\right) \vee\left(Y, \star ; \Sigma_{Y}, \mathcal{B}_{2}\right) \stackrel{\text { def }}{=} Q\left(\left(X, \star ; \Sigma_{X}, \mathcal{B}_{1}\right) \cup\left(Y, \star ; \Sigma_{Y}, \mathcal{B}_{2}\right)\right) .
$$

- We define the product

$$
\left(X, \star ; \Sigma_{X}, \mathcal{B}_{1}\right) \wedge\left(Y, \star ; \Sigma_{Y}, \mathcal{B}_{2}\right) \stackrel{\text { def }}{=} Q\left(\left(X, \star ; \Sigma_{X}, \mathcal{B}_{1}\right) \times\left(Y, \star ; \Sigma_{Y}, \mathcal{B}_{2}\right)\right) .
$$

There is an important relation between the quotient spaces induced by the sum of $\Sigma$-disjoint pairs.

Proposition 2.16. Let $\left(X ; \Sigma_{X}, \mathcal{B}\right)$ be a space with symmetries, let $(A, B)$ and $(C, D)$ be pairs of subsets of $X$ such that $A$ and $C$ are $\Sigma$-disjoint. Then the spaces

$$
Q\left(\left(A, B ; \Sigma_{A}, \mathcal{B}\right) \cup\left(C, D ; \Sigma_{C}, \mathcal{B}\right)\right) \text { and } Q\left(A, B ; \Sigma_{A}, \mathcal{B}\right) \vee Q\left(C, D ; \Sigma_{C}, \mathcal{B}\right)
$$

are $\Sigma$-homeomorphic.

ProOF. We consider the canonical projections

$$
p_{A}: A \rightarrow A / B \vee C / D, p_{C}: C \rightarrow A / B \vee C / D, p_{A \cup C}: A \cup C \rightarrow A \cup C / B \cup D
$$

and the homeomorphism

$$
h: A \cup C / B \cup D \rightarrow A / B \vee C / D, h(x)= \begin{cases}x & \text { if } x \neq B \cup D \\ \{B, D\} & \text { if } x=B \cup D .\end{cases}
$$

In order to prove that $h$ is a $\Sigma$-homeomorphism we observe the following. 
Let $a \in \Sigma_{A \cup C / B \cup D}$ and $|a| \neq\{\star\}$. Then there is $b \in \Sigma_{A \cup B}=\Sigma_{A} \cup \Sigma_{B}$ such that $p_{A \cup B}(|b|)=|a|$ and $(b)=(a)$. Thus, $h(|a|)=p_{A}(|b|)$ when $b \in \Sigma_{A}$ or $h(|a|)=p_{B}(|b|)$ when $b \in \Sigma_{B}$.

Let $a \in \Sigma_{A / B \vee C / D}$ and $|a| \neq\{\star\}$. Then there is either $b \in \Sigma_{A} \subset \Sigma_{A \cup C}$ or $c \in \Sigma_{C} \subset$ $\Sigma_{A \cup C}$ such that either $p_{A}(|b|)=|a|,(b)=(a)$ or $p_{B}(|c|)=|a|,(c)=(a)$. Thus, either $h^{-1}(|a|)=p_{A \cup C}(|b|)$ or $h^{-1}(|a|)=p_{A \cup C}(|c|)$.

The following propositions describe the relation between pointed symmetries and the operations of sum and product.

Proposition 2.17. Let $\left(X, \star, \Sigma_{X}, \mathcal{B}_{1}\right)$ and $\left(Y, \star ; \Sigma_{Y}, \mathcal{B}_{2}\right)$ be spaces with symmetries. Then

(1) $\left(\left(X, \star ; \Sigma_{X}, \mathcal{B}_{1}\right) \vee\left(Y, \star ; \Sigma_{Y}, \mathcal{B}_{2}\right)\right)^{\star}=\left(X, \star ; \Sigma_{X}, \mathcal{B}_{1}\right)^{\star} \vee\left(Y, \star ; \Sigma_{Y}, \mathcal{B}_{2}\right)^{\star}$ if the spaces $X$ and $Y$ are disjoint.

(2) $\left(\left(X, \star ; \Sigma_{X}, \mathcal{B}_{1}\right) \wedge\left(Y, \star ; \Sigma_{Y}, \mathcal{B}_{2}\right)\right)^{\star}=\left(X, \star ; \Sigma_{X}, \mathcal{B}_{1}\right)^{\star} \wedge\left(Y, \star ; \Sigma_{Y}, \mathcal{B}_{2}\right)^{\star}$.

Proof. (1) Let $(X \vee Y, \Sigma, \mathcal{B})=\left(\left(X, \star ; \Sigma_{X}, \mathcal{B}_{1}\right) \vee\left(Y, \star ; \Sigma_{Y}, \mathcal{B}_{2}\right)\right)^{\star}$ and let $p: X \cup Y \rightarrow$ $X \vee Y$ be the canonical projection. Here, $\star$ stands for the base points in $X, Y$ or $X \vee Y$.

We notice that $a \in \Sigma$ if and only if $|a|=\{\star\}$ or $a=(p(|b| \cup\{\star\}),(b))$ for some $b \in \Sigma_{X} \cup \Sigma_{Y}$ and $a \in \Sigma_{X \vee Y}^{\star}$ if and only if $|a|=\{\star\}, a=(p(|b|) \cup\{\star\},(b))$ for some $b \in \Sigma_{X} \cup \Sigma_{Y}$.

But $p(|b| \cup\{\star\})=p(|b|) \cup\{\star\}$ for any $b \in \Sigma_{X} \cup \Sigma_{Y}$. This shows how the elements of the symmetries are related.

(2) Let $p: X \times Y \rightarrow X \wedge Y$ be the canonical projection and let $a \in \Sigma_{X}, b \in \Sigma_{Y}$. We observe

$$
\begin{aligned}
p((|a| \cup\{\star\}) \times(|b| \cup\{\star\})) & =p(|a| \times|b| \cup|a| \times\{\star\} \cup\{\star\} \times|b| \cup\{\star\} \times\{\star\}) \\
& =p(|a| \times|b|) \cup\{\star\}
\end{aligned}
$$

This shows how the elements of the symmetries can be obtained from one another.

2.3.3. Special maps on quotient spaces of pairs of sets. The construction of the Conley index is founded on the properties of maps on spaces with base point of a special form. In this section we study the properties of such maps in relation to symmetries.

We consider spaces $\left(X ; \Sigma_{X}, \mathcal{B}\right),\left(Y ; \Sigma_{Y}, \mathcal{B}\right)$ and an invariant map $F:\left(X ; \Sigma_{X}, \mathcal{B}\right) \rightarrow$ $\left(Y ; \Sigma_{Y}, \mathcal{B}\right)$. For given sets $A, B, C \subset X$ and $D, E \subset Y$ such that

1. $B \subset A \subset X$,

2. $E \subset D \subset Y$,

3. $C \subset(A \backslash B)$ and $F(C) \subset(D \backslash E)$,

we consider a map

$$
f:(A / B, \star) \rightarrow(D / E, \star), f(p(x))= \begin{cases}p(F(x)) & \text { if } p(x) \in C \\ \star & \text { if } p(x) \notin C,\end{cases}
$$

where $p: A \rightarrow A / B$ and $p: D \rightarrow D / E$ denote the canonical projections. We additionally assume that $f$ is continuous, which does not hold for arbitrary sets $A, \ldots, E$.

Proposition 2.18. The map $f: Q^{\star}\left(A, B ; \Sigma_{A}, \mathcal{B}\right) \rightarrow Q^{\star}\left(D, E ; \Sigma_{D}, \mathcal{B}\right)$ preserves the weak quotient symmetries. 
Proof. Let $a \in \Sigma_{A / B}^{\star}$. If $|a|=\{\star\}$ then $(\{f(\star)\},(a))=(\{\star\},(a)) \in \Sigma_{D / E}^{\star}$. If $|a| \neq\{\star\}$ then there must exist $b \in \Sigma_{X}$ such that $a=(p(|b| \cap A) \cup\{\star\},(b))$. We know that $F(|b|) \subset|c|$ and $(c)=(a)$ for some $c \in \Sigma_{Y}$. Hence

$$
\begin{aligned}
f(|a|) & =f(p(|b \cap A|) \cup\{\star\})=f(p(|b \cap A|)) \cup\{\star\} \\
& =[f(p(|b| \cap C)) \cup f(p(|b| \cap(A \backslash C)))] \cup\{\star\} \\
& =[p(F(|b| \cap C)) \cup\{\star\}] \cup\{\star\} \subset p(|c| \cap D) \cup\{\star\}
\end{aligned}
$$

and $(p(|c| \cap D) \cup\{\star\},(a)) \in \Sigma_{D / E}^{\star}$.

Proposition 2.19. If $C$ is a $\Sigma$-invariant subset of $X$, i.e. $C \in P_{\Sigma_{X}}(X)$, then the map $f: Q\left(A, B ; \Sigma_{A}, \mathcal{B}\right) \rightarrow Q\left(D, E ; \Sigma_{D}, \mathcal{B}\right)$ preserves the quotient symmetries.

Proof. Let $a \in \Sigma_{A / B}$. If $|a|=\{\star\}$ then $(\{f(\star)\},(a))=(\{\star\},(a)) \in \Sigma_{D / E}$. If $|a| \neq\{\star\}$ then there must exist $b \in \Sigma_{X}$ such that $a=(p(|b| \cap A),(b))$. Hence $|b| \cap C=$ $\emptyset$ or $|b| \subset C$ as $C$ is $\Sigma_{X}$-invariant. Thus, when $|b| \cap C=\emptyset$, we have

$$
(f(|a|),(a))=(\{\star\},(a)) \in \Sigma_{D / E} .
$$

If $|b| \subset C$, then $F(|b|) \subset|c|$ and $(c)=(a)$ for some $c \in \Sigma_{Y}$, so

$$
f(|a|)=p(F(|b|)) \subset p(|c| \cap D)
$$

and $(p(|c| \cap D),(a)) \in \Sigma_{D / E}$.

2.4. Homotopy type of topological spaces with base point and symmetries. Maps $f, g$ : $\left(X, \star ; \Sigma_{X}, \mathcal{B}\right) \rightarrow\left(Y, \star ; \Sigma_{Y}, \mathcal{B}\right)$ are $\Sigma$-homotopy equivalent, written $f \stackrel{\Sigma}{\sim} g$, if there exists a map $H: X \times[0,1] \rightarrow Y$ such that

1. $H_{t}=H(\cdot, t):\left(X, \star ; \Sigma_{X}, \mathcal{B}\right) \rightarrow\left(Y, \star ; \Sigma_{Y}, \mathcal{B}\right)$,

2. $H_{0}=f$ and $H_{1}=g$.

A map $f:\left(X, \star ; \Sigma_{X}, \mathcal{B}\right) \rightarrow\left(Y, \star ; \Sigma_{Y}, \mathcal{B}\right)$ is a $\Sigma$-homotopy equivalence if there is a map $g:\left(Y, \star ; \Sigma_{Y}, \mathcal{B}\right) \rightarrow\left(X, \star ; \Sigma_{X}, \mathcal{B}\right)$ such that $f \circ g \stackrel{\Sigma}{\sim} i d_{Y}$ and $g \circ f \stackrel{\Sigma}{\sim} i d_{X}$.

The spaces $\left(X, \star ; \Sigma_{X}, \mathcal{B}\right)$ and $\left(Y, \star ; \Sigma_{Y}, \mathcal{B}\right)$ with symmetries are $\Sigma$-homotopy equivalent if there exists a $\Sigma$-homotopy equivalence $f:\left(X, \star ; \Sigma_{X}, \mathcal{B}\right) \rightarrow\left(Y, \star ; \Sigma_{Y}, \mathcal{B}\right)$.

This leads to the notion of the $\Sigma$-homotopy type of $\left(X, \star ; \Sigma_{X}, \mathcal{B}\right)$ which will be denoted by $\left[\left(X, \star ; \Sigma_{X}, \mathcal{B}\right)\right]$.

Remark 2.13 leads directly to

Remark 2.20. If $\left[\left(X, \star ; \Sigma_{X}, \mathcal{B}\right)\right]=\left[\left(Y, \star ; \Sigma_{Y}, \mathcal{B}\right)\right]$, then $\left[\left(X, \star ; \Sigma_{X}^{\star}, \mathcal{B}\right)\right]=\left[\left(Y, \star ; \Sigma_{Y}^{\star}, \mathcal{B}\right)\right]$.

Standard properties of sum and product of spaces with base point (see for instance [Wh, chapter III.2]) show that there are well defined operations of sum and product of $\Sigma$-homotopy types of spaces.

Definition 2.21. Let $\mathcal{X}=\left[\left(X, \star ; \Sigma_{X}, \mathcal{B}\right)\right]$ and $\mathcal{Y}=\left[\left(Y, \star ; \Sigma_{Y}, \mathcal{B}\right)\right]$ be $\Sigma$-homotopy types of spaces with symmetries. We define

- the sum of $\mathcal{X}$ and $\mathcal{Y}$ by

$$
\mathcal{X} \vee \mathcal{Y} \stackrel{\text { def }}{=}\left[\left(X, \star ; \Sigma_{X}, \mathcal{B}\right) \vee\left(Y, \star ; \Sigma_{Y}, \mathcal{B}\right)\right],
$$


- the product of $\mathcal{X}$ and $\mathcal{Y}$ by

$$
\mathcal{X} \wedge \mathcal{Y} \stackrel{\text { def }}{=}\left[\left(X, \star ; \Sigma_{X}, \mathcal{B}\right) \wedge\left(Y, \star ; \Sigma_{Y}, \mathcal{B}\right)\right] .
$$

3. Examples of generalized symmetries. In this section we describe some examples of generalized symmetries which appear in a natural way in examining some classes of flows.

3.1. Generalized symmetries induced by a group action. Let $G$ be a topological group, which is not assumed to be compact. Let $\mathcal{B}=S(G)$, the basic set of the symmetries, be the family of closed subgroups of $G$. Moreover let the group $G$ act on a space $X$. Then we define

$$
\Sigma_{G}(X) \stackrel{\text { def }}{=}\left\{\left(G x, G_{x}\right): x \in X\right\}, \quad \widehat{\Sigma}_{G}(X) \stackrel{\text { def }}{=}\left\{(G x, H): x \in X, H \subset G_{x}\right\} .
$$

where $G x=\{g x \in X: g \in G\}$ and $G_{x}=\{g \in G: g x=x\}$ for any $x \in X$.

Elementary verification leads to the following statements.

Remark 3.1. A subset $A \subset X$ is $G$-invariant if and only if it is $\Sigma_{G}(X)$-invariant.

REMARK 3.2. If $G$ is compact then the symmetries $\Sigma_{G}(X)$ and $\widehat{\Sigma}_{G}(X)$ are regular.

Proposition 3.3. If $f:(X, G) \rightarrow(Y, G)$ is a G-equivariant homeomorphism then

$$
f:\left(X ; \Sigma_{G}(X)\right) \rightarrow\left(Y ; \Sigma_{G}(Y)\right)
$$

preserves the symmetries.

Proposition 3.4. If $f:(X, G) \rightarrow(Y, G)$ is a $G$-equivariant map then

$$
f:\left(X ; \widehat{\Sigma}_{G}(X)\right) \rightarrow\left(Y ; \widehat{\Sigma}_{G}(Y)\right)
$$

preserves the symmetries.

The above remarks suggest some kinds of symmetries which can be considered in the case when the flow examined preserves an action of a topological group.

It also suggests the relations between the $G$-homotopy type of the $G$-spaces and the $\Sigma$-homotopy type of the spaces with the induced symmetries.

3.2. Generalized symmetries induced by isolated invariant sets of a flow. We consider a flow $\psi: X \times \mathbf{R} \rightarrow X$ and we introduce the symmetries induced by the compact isolated invariant sets $I I S(\psi)$ of the flow $\psi$, see section 5.1. Let

$$
\mathcal{B}=I I S(\psi) \times I I S(\psi)
$$

be the basic set of the symmetries.

We define

$$
\Sigma \stackrel{\text { def }}{=}\left\{(\psi(\{x\} \times \mathbf{R}),(A, B)): x \in X,(A, B) \in \mathcal{B}, \alpha_{\psi}(x) \cap A \neq \emptyset, \omega_{\psi}(x) \cap B \neq \emptyset\right\},
$$

where $\alpha_{\psi}(\cdot)$ and $\omega_{\psi}(\cdot)$ denote the negative and positive limit sets of the flow $\psi$ respectively.

The family $\Sigma$ describes the connecting trajectories of the flow $\psi$. 
We prove that any flow $\varphi: X \times \mathbf{R} \rightarrow X$ which commutes with $\psi$, i.e. $\varphi^{t} \circ \psi^{s}=$ $\psi^{s} \circ \varphi^{t}$ for $t, s \in \mathbf{R}$, preserves the symmetries $\Sigma$.

Lemma 3.5. Let $A \in I I S(\psi)$. Then $\varphi^{t}(A) \subset A$ for any $t \in \mathbf{R}$.

Proof. The assumption guarantees the existence of a compact subset $N \subset X$ such that $A=\operatorname{inv}(N, \psi) \subset \operatorname{int}(N)$. As $A$ is compact and the flow $\varphi$ is continuous there is $\eta>0$ such that $\varphi(A \times[-\eta, \eta]) \subset \operatorname{int}(N)$. Hence, for any $x \in A, t \in[-\eta, \eta]$ and $s \in \mathbf{R}$, $\psi^{s}\left(\varphi^{t}(x)\right)=\varphi^{t}\left(\psi^{s}(x)\right) \in \operatorname{int}(N) \subset N$, so we finally obtain $\varphi^{t}(x) \in \operatorname{inv}(N, \psi)=A$.

Corollary 3.6. Let $A \in I I S(\psi)$ and $x \in X$ satisfy $\omega_{\psi}(x) \cap A \neq \emptyset$. Then $\omega_{\psi}\left(\varphi^{t}(x)\right)$ $\cap A \neq \emptyset$ for any $t \in \mathbf{R}$.

PROOF. Indeed, if $\lim _{n \rightarrow \infty} \psi^{t_{n}}(x)=x_{0} \in A$ for some sequence $t_{n} \rightarrow+\infty$ then

$$
\lim _{n \rightarrow \infty} \psi^{t_{n}}\left(\varphi^{t}(x)\right)=\lim _{n \rightarrow \infty} \varphi^{t}\left(\psi^{t_{n}}(x)\right)=\varphi^{t}\left(x_{0}\right) \in A
$$

for any $t \in \mathbf{R}$.

Proposition 3.7. For any $t \in \mathbf{R}, \varphi^{t}:(X ; \Sigma, \mathcal{B}) \rightarrow(X ; \Sigma, \mathcal{B})$ preserves the symmetries.

Proof. For any $x \in X$ and $t \in \mathbf{R}$ we obtain $\varphi^{t}(\psi(\{x\} \times \mathbf{R}))=\psi\left(\left\{\varphi^{t}(x)\right\} \times \mathbf{R}\right)$, as the flows $\varphi$ and $\psi$ commute. Hence, Corollary 3.6 completes the proof.

3.3. Generalized symmetries for flows admitting a first integral. Let $f: X \rightarrow \mathbf{R}$ be a given function. We define $\mathcal{B}=\mathbf{R}$ to be the basic set of the symmetries

$$
\Sigma(f)=\left\{\left(f^{-1}(c), c\right): c \in f(X)\right\} .
$$

We consider a flow $\varphi: X \times \mathbf{R} \rightarrow X$ which admits $f: X \rightarrow \mathbf{R}$ as a first integral, i.e. $f=f \circ \varphi^{t}$ for any $t \in \mathbf{R}$.

Proposition 3.8. For $t \in \mathbf{R}, \varphi^{t}:(X ; \Sigma(f), \mathcal{B}) \rightarrow(X ; \Sigma(f), \mathcal{B})$ preserves the symmetries.

This example can be extended if one replaces $\mathbf{R}$ with an arbitrary space $Y$, takes $\mathcal{B} \subset P(Y)$ and

$$
\Sigma(f)=\left\{\left(f^{-1}(A), A\right): A \in \mathcal{B}, A \cap f(X) \neq \emptyset\right\} .
$$

Such symmetries might be helpful for examining the properties of flows which admit first integrals or invariant subspaces in a more general situation.

4. Some examples of spaces with generalized symmetries. Now, we present some simple examples of spaces with generalized symmetries. We will use them in section 6 to show how the Conley index with symmetries can be applied to examine the behaviour of flows.

Let us consider the following subsets of the Euclidean space $\left(\mathbf{R}^{2},|\cdot|\right)$ :

- discs:

$$
\begin{gathered}
D_{1}=\left\{x \in \mathbf{R}^{2}:|x-(3,0)| \leqslant 3\right\}, \\
D_{2}=\left\{x \in \mathbf{R}^{2}:|x-(-2,0)| \leqslant 2\right\}, D_{3}=\left\{x \in \mathbf{R}^{2}:|x-(-2,0)| \leqslant 1\right\},
\end{gathered}
$$


- circles:

$$
E_{1}=\left\{x \in \mathbf{R}^{2}:|x-(2,0)|=2\right\}, E_{2}=\left\{x \in \mathbf{R}^{2}:|x-(1,0)|=1\right\},
$$

- points:

$$
P_{1}=(0,0), P_{2}=(3,0), P_{3}=(-2,0), P_{4}=(-1,0) .
$$

We define a space with generalized symmetries over the base set

$$
\mathcal{B}=\{1,2,3\} \subset \mathbf{N} .
$$

This will be done, roughly speaking, in the following way. The circles $E_{1}, E_{2}$ will define the elements of symmetries $\left(E_{1}, 1\right)$ and $\left(E_{2}, 1\right)$ of type 1 . The points $P_{2}, P_{3}$ will determine the elements $\left(P_{2}, 2\right)$ and $\left(P_{3}, 2\right)$ of type 2 . The points $P_{1}$ and $P_{4}$ will play the role of base points in suitable spaces, so by the definition of symmetries in spaces with base point, they will appear with types 1,2,3. Moreover we will introduce elements of type 3 which will take the form $(\{x, \star\}, 3)$, where $x$ will belong to an appropriate set and $\star$ is a base point.

We define the following spaces.

- $\left(U, \star ; \Sigma_{U}\right)=\left(D_{1}, P_{1} ; \Sigma_{U}\right)$, where

$$
\begin{aligned}
\Sigma_{U}= & \left\{\left(E_{1}, 1\right),\left(\left\{P_{2}, P_{1}\right\}, 2\right)\right\} \\
& \cup\left\{\left(\left\{x, P_{1}\right\}, 3\right): x \in D_{1} \backslash\left(E_{1} \cup\left\{P_{2}\right\}\right)\right\} \\
& \cup\left\{\left(\left\{P_{1}\right\}, 1\right),\left(\left\{P_{1}\right\}, 2\right),\left(\left\{P_{1}\right\}, 3\right)\right\}
\end{aligned}
$$

- $\left(W, \star ; \Sigma_{W}\right)=\left(D_{1}, P_{1} ; \Sigma_{W}\right)$, where

$$
\begin{aligned}
\Sigma_{W}= & \left\{\left(E_{2}, 1\right),\left(\left\{P_{2}, P_{1}\right\}, 2\right)\right\} \\
& \cup\left\{\left(\left\{x, P_{1}\right\}, 3\right): x \in D_{1} \backslash\left(E_{2} \cup\left\{P_{2}\right\}\right)\right\} \\
& \cup\left\{\left(\left\{P_{1}\right\}, 1\right),\left(\left\{P_{1}\right\}, 2\right),\left(\left\{P_{1}\right\}, 3\right)\right\}
\end{aligned}
$$

- $\left(X, \star ; \Sigma_{X}\right)=\left(D_{1} \cup D_{2}, P_{1} ; \Sigma_{X}\right)$, where

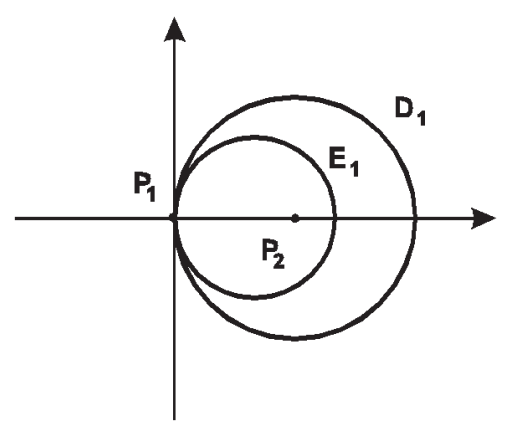

$$
\begin{aligned}
\Sigma_{X}=\left\{\left(E_{2}, 1\right),\left(\left\{P_{3}, P_{1}\right\}, 2\right)\right\} \\
\cup\left\{\left(\left\{x, P_{1}\right\}, 3\right): x \in\left(D_{1} \cup D_{2}\right) \backslash\left(E_{2} \cup\left\{P_{3}\right\}\right)\right\} \\
\cup\left\{\left(\left\{P_{1}\right\}, 1\right),\left(\left\{P_{1}\right\}, 2\right),\left(\left\{P_{1}\right\}, 3\right)\right\}
\end{aligned}
$$

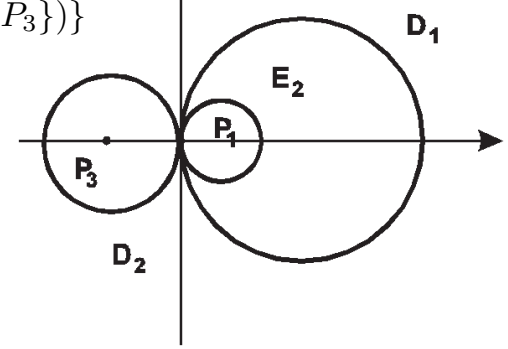

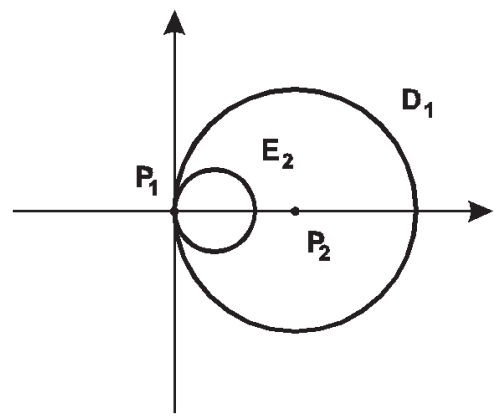


- $\left(Y, \star ; \Sigma_{Y}\right)=\left(D_{1}, P_{1} ; \Sigma_{Y}\right)$, where

$$
\begin{aligned}
\Sigma_{Y}= & \left\{\left(E_{2}, 1\right)\right\} \\
& \cup\left\{\left(\left\{x, P_{1}\right\}, 3\right): x \in D_{1} \backslash E_{2}\right\} \\
& \cup\left\{\left(\left\{P_{1}\right\}, 1\right),\left(\left\{P_{1}\right\}, 2\right),\left(\left\{P_{1}\right\}, 3\right)\right\}
\end{aligned}
$$

- $\left(Z, \star ; \Sigma_{Z}\right)=\left(D_{3}, P_{4} ; \Sigma_{Z}\right)$, where

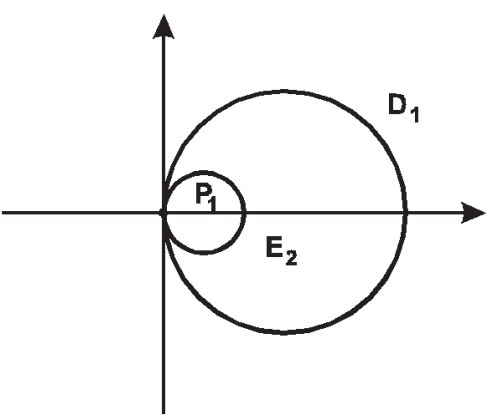

$$
\begin{aligned}
\Sigma_{Z}= & \left\{\left(\left\{P_{3}, P_{4}\right\}, 2\right)\right\} \\
& \cup\left\{\left(\left\{x, P_{4}\right\}, 3\right): x \in D_{3} \backslash\left\{P_{3}, P_{4}\right\}\right\} \\
& \cup\left\{\left(\left\{P_{4}\right\}, 1\right),\left(\left\{P_{4}\right\}, 2\right),\left(\left\{P_{4}\right\}, 3\right)\right\}
\end{aligned}
$$

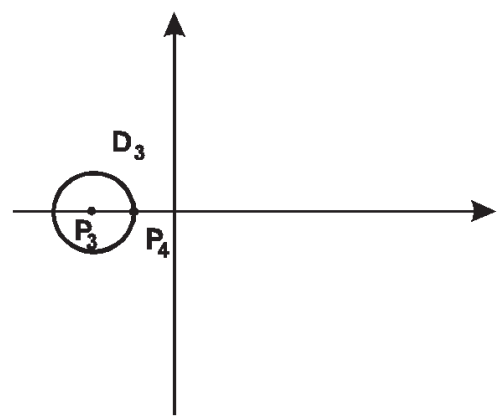

The definition of the sum of spaces with symmetries leads us to the following remark.

REMARK 4.1. The spaces $\left(X, \star ; \Sigma_{Y}\right)$ and $\left(Y, \star ; \Sigma_{Y}\right) \vee\left(Z, \star ; \Sigma_{Z}\right)$ are $\Sigma$-homeomorphic.

REMARK 4.2. The spaces $\left(X, \star ; \Sigma_{X}\right)$ and $\left(W, \star ; \Sigma_{W}\right)$ are $\Sigma$-homotopy equivalent.

Lemma 4.3. The spaces $\left(U, \star ; \Sigma_{U}\right)$ and $\left(W, \star ; \Sigma_{W}\right)$ are not $\Sigma$-homotopy equivalent.

Proof. Assume that $\left(U, \star ; \Sigma_{U}\right)$ and $\left(W, \star ; \Sigma_{W}\right)$ are $\Sigma$-homotopy equivalent. Hence, there exist $f:\left(U, \star ; \Sigma_{U}\right) \rightarrow\left(W, \star ; \Sigma_{W}\right), g:\left(W, \star ; \Sigma_{W}\right) \rightarrow\left(U, \star ; \Sigma_{U}\right)$ and a $\Sigma$-homotopy $H:(U, \star) \times[0,1] \rightarrow(U, \star)$ which connects $g \circ f$ to $i d_{U}$.

This leads to the following observations:

- $f\left(E_{1}\right) \subset E_{2}, f\left(P_{2}\right)=P_{2}$, and $f^{-1}\left(P_{2}\right)=\left\{P_{2}\right\}$,

- $g\left(E_{2}\right) \subset E_{1}, g\left(P_{2}\right)=P_{2}$, and $g^{-1}\left(P_{2}\right)=\left\{P_{2}\right\}$,

- $H_{s}\left(E_{1}\right) \subset E_{1}, H_{s}\left(P_{2}\right)=P_{2}$, and $H_{s}^{-1}\left(P_{2}\right)=\left\{P_{2}\right\}$, for any $s \in[0,1]$.

Hence, for $\widetilde{U} \stackrel{\text { def }}{=} U \backslash\left\{P_{2}\right\}$ and $\widetilde{W} \stackrel{\text { def }}{=} W \backslash\left\{P_{2}\right\}$, there are well defined maps

$$
\widetilde{f} \stackrel{\text { def }}{=} f_{\mid \widetilde{U}}:(\widetilde{U}, \star) \rightarrow(\widetilde{W}, \star), \quad \widetilde{g} \stackrel{\text { def }}{=} g_{\mid \widetilde{U}}:(\widetilde{W}, \star) \rightarrow(\widetilde{U}, \star),
$$

and the homotopy

connects $\widetilde{g} \circ \widetilde{f}$ to $i d_{\widetilde{U}}$.

$$
\widetilde{H} \stackrel{\text { def }}{=} H_{\mid \widetilde{U} \times[0,1]}:(\widetilde{U}, \star) \times[0,1] \rightarrow(\widetilde{U}, \star)
$$

We note that

$$
\gamma:[0,1] \rightarrow E_{1}, \gamma(t)=(2-2 \cdot \cos 2 \pi t, 2 \cdot \sin 2 \pi t)
$$

which is a parametrization of the circle $E_{1}$, represents a nontrivial element $[\gamma]$ in the fundamental group $\pi_{1}(\widetilde{U}, \star)$. 
On the other hand $f\left(E_{1}\right) \subset E_{2}$, so $[\tilde{f} \circ \gamma]$ is the trivial element of $\pi_{1}(\widetilde{W}, \star)$. Hence $[\widetilde{g} \circ \widetilde{f} \circ \gamma]$ is the trivial element of $\pi_{1}(\widetilde{U}, \star)$. This contradicts $[\gamma]=[\widetilde{g} \circ \widetilde{f} \circ \gamma]$ as $\widetilde{g} \circ \widetilde{f}$ and $i d_{\widetilde{U}}$ are homotopy equivalent.

5. Homotopy index for flows preserving generalized symmetries. In this section we consider a locally compact metric space with symmetries $\left(X ; \Sigma_{X}, \mathcal{B}\right)$ and a flow $\varphi$ : $X \times \mathbf{R} \rightarrow X$ which preserves the symmetries, i.e. $\varphi^{t}=\varphi(\cdot, t):\left(X ; \Sigma_{X}, \mathcal{B}\right) \rightarrow\left(X ; \Sigma_{X}, \mathcal{B}\right)$ for any $t \in \mathbf{R}$.

5.1. General definitions. Firstly we recall the definitions of objects studied in Conley index theory and formulate additional conditions which are related to the presence of symmetries.

Let us recall that for any subset $A \subset X$ we call

$$
i n v(A)=i n v(A, \varphi) \stackrel{\text { def }}{=} \bigcap_{t \in \mathbf{R}} \varphi^{-t}(A)
$$

the invariant part of $A$.

The subset $A \subset X$ is an isolating neighbourhood if

$$
\operatorname{inv}(\operatorname{cl}(A), \varphi) \subset \operatorname{int}(A) .
$$

A subset $S \subset X$ is an isolated invariant set of the flow $\varphi, S \in I I S(\varphi)$, if there exists a compact isolating neighbourhood $A \subset X$ such that

$$
S=\operatorname{inv}(A, \varphi) .
$$

A pair $(N, L)$ of compact subsets $L \subset N \subset X$ is an index pair when the following conditions are satisfied:

1. $N \backslash L$ is an isolating neighbourhood,

2. $L$ is an exit set for the subset $N$ :

$$
\forall_{x \in N} \forall_{t>0} \exists_{\tau \in(0, t)} \varphi^{t}(x) \notin N \Rightarrow \varphi^{\tau}(x) \in L,
$$

3. $L$ is positively invariant in $N$ :

$$
\forall_{x \in L} \forall_{t>0} \varphi^{[0, t]}(x) \subset N \Rightarrow \varphi^{[0, t]}(x) \subset L .
$$

Moreover, the index pair $(N, L)$ is regular if the exit time mapping

$$
\tau: N \rightarrow[0,+\infty], \tau(x)= \begin{cases}\sup \{t>0: \varphi(\{x\} \times[0, t]) \subset N \backslash L\} & \text { if } x \in N \backslash L \\ 0 & \text { if } x \in L\end{cases}
$$

is continuous.

Finally, the index pair $(N, L)$ is an index pair of the set $S$ if $S=\operatorname{inv}(N \backslash L, \varphi)$. Hence, of course, $S$ is a compact isolated invariant set of the flow $\varphi$.

The fundamental theorem of Conley index theory is

THEOREM 5.1. If $A$ is an isolating neighbourhood then there exists a regular index pair $(N, L)$ of the set inv $(A)$ such that $N \subset A$.

For the proof we refer the reader to [RS], theorem (5.2), and [S], theorems (5.2-4).

In our case with symmetries we introduce the following definitions. 
Definition 5.2. A (regular) index pair $(N, L)$ is a $\Sigma$-invariant (regular) index pair if the sets $N, L$ are $\Sigma$-invariant subsets of $X$.

Definition 5.3. A subset $S \subset X$ is a $\Sigma$-admissible compact isolated invariant set of the flow $\varphi, S \in I I S_{\Sigma}(\varphi)$, if it admits a $\Sigma$-invariant index pair.

Definition 5.4. Sets $S_{1}, S_{2} \in \operatorname{IIS}(\varphi)$ are said to be $(\Sigma, \varphi)$-disjoint if they admit index pairs $\left(N_{1}, L_{1}\right)$ and $\left(N_{1}, L_{1}\right)$ respectively such that the subsets $N_{1}$ and $N_{2}$ are $\Sigma$-disjoint.

In section 5.4 we prove that when the symmetries $\Sigma$ are regular then a set $S \in I I S(\varphi)$ is $\Sigma$-admissible if and only if it is $\Sigma$-invariant, and two $\Sigma$-admissible sets $S_{1}, S_{2} \in \operatorname{IIS}(\varphi)$ are $(\Sigma, \varphi)$-disjoint if and only if they are disjoint.

5.2. Homotopy index with symmetries. In this section we introduce the homotopy index of a compact isolated invariant set of a flow which reflects the structure of symmetries.

We consider a flow $\varphi:\left(X ; \Sigma_{X}, \mathcal{B}\right) \times \mathbf{R} \rightarrow\left(X ; \Sigma_{X}, \mathcal{B}\right)$ which preserves the symmetries and a compact isolated invariant set $S \in I I S(\varphi)$.

In this situation, two cases can appear according to whether $S$ is $\Sigma$-admissible, i.e. has a $\Sigma$-invariant index pair, or not. Usually, invariant theories deal with somehow distinguished invariant objects. We show that both cases can be successfully treated. This is a great difference in comparison to the usually proposed invariant index theory.

Definition 5.5. For any set $S \in I I S(\varphi)$ we define the homotopy $\Sigma^{\star}$-Conley index

$$
\Sigma^{\star} h(S, \varphi) \stackrel{\text { def }}{=}\left[Q^{\star}\left(N, L ; \Sigma_{N}, \mathcal{B}\right)\right]
$$

where $(N, L)$ is an arbitrary index pair of $S$.

In the case when the set $S \in I I S(\varphi)$ is additionally $\Sigma$-admissible, i.e. it admits a $\Sigma$-invariant index pair, we can describe a more precise homotopy index.

Definition 5.6. For any $\Sigma$-admissible set $S \in I I S_{\Sigma}(\varphi)$ we define the homotopy $\Sigma$ Conley index

$$
\Sigma h(S, \varphi) \stackrel{\text { def }}{=}\left[Q\left(N, L ; \Sigma_{N}, \mathcal{B}\right)\right]
$$

where $(N, L)$ is an arbitrary $\Sigma$-invariant index pair of $S$.

The independence of these indexes from the choice of index pairs follows from the standard arguments used in the Conley index theory. We recall the basic constructions and refer the reader to D. Salamon [S] and J. Robbin \& D. Salamon [RS].

Let us consider two index pairs $\left(N_{\alpha}, L_{\alpha}\right)$ and $\left(N_{\beta}, L_{\beta}\right)$ of $S \in I I S(\varphi)$. For $t \geqslant 0$ we define

$$
\begin{aligned}
C_{\beta \alpha}^{t} & \stackrel{\text { def }}{=}\left\{x \in N_{\alpha} \backslash L_{\alpha}: \varphi^{\left[0, \frac{2 t}{3}\right]}(x) \subset N_{\alpha} \backslash L_{\alpha}, \varphi^{\left[\frac{t}{3}, t\right]}(x) \subset N_{\beta} \backslash L_{\beta}\right\} \\
& =\bigcap_{\tau \in\left[0, \frac{2 t}{3}\right]} \varphi^{-\tau}\left(N_{\alpha} \backslash L_{\alpha}\right) \cap \bigcap_{\tau \in\left[\frac{t}{3}, t\right]} \varphi^{-\tau}\left(N_{\beta} \backslash L_{\beta}\right)
\end{aligned}
$$

and

$$
f_{\beta \alpha}^{t}: N_{\alpha} / L_{\alpha} \rightarrow N_{\beta} / L_{\beta}, f_{\beta \alpha}^{t}(p(x))= \begin{cases}p\left(\varphi^{t}(x)\right) & \text { if } x \in C_{\beta \alpha}^{t}, \\ \star & \text { if } x \notin C_{\beta \alpha}^{t},\end{cases}
$$

where $p: N_{\alpha} \rightarrow N_{\alpha} / L_{\alpha}$ and $p: N_{\beta} \rightarrow N_{\beta} / L_{\beta}$ are the canonical projections. 
Moreover, let

$$
F_{\alpha}: N_{\alpha} / L_{\alpha} \times[0,+\infty) \rightarrow N_{\alpha} / L_{\alpha}, F(x, t)=f_{\alpha \alpha}^{t}(x) .
$$

We recall the fundamental properties of the maps defined above. They are proved in detail by J. Robbin and D. Salamon [RS], theorem (6.3) and theorem (4.2).

TheOREM 5.7. There is $T_{\beta \alpha} \geqslant 0$ such that the maps $f_{\beta \alpha}^{t}: N_{\alpha} / L_{\alpha} \rightarrow N_{\beta} / L_{\beta}$ and $f_{\alpha \beta}^{t}: N_{\beta} / L_{\beta} \rightarrow N_{\alpha} / L_{\alpha}$ are continuous for any $t \geqslant T_{\beta \alpha}$ and

$$
f_{\alpha \alpha}^{2 t}=f_{\alpha \beta}^{t} \circ f_{\beta \alpha}^{t}, f_{\beta \beta}^{2 t}=f_{\beta \alpha}^{t} \circ f_{\alpha \beta}^{t} .
$$

TheOREM 5.8. The map $F_{\alpha}: N_{\alpha} / L_{\alpha} \times[0,+\infty) \rightarrow N_{\alpha} / L_{\alpha}$ is continuous.

Now, we apply this technique to prove the correctness of the definitions of Conley indexes with symmetries.

Lemma 5.9. For $t>T_{\beta \alpha}$, the map $f_{\beta \alpha}^{t}: Q^{\star}\left(N_{\alpha}, L_{\alpha} ; \Sigma_{N_{\alpha}}, \mathcal{B}\right) \rightarrow Q^{\star}\left(N_{\beta}, L_{\beta} ; \Sigma_{N_{\beta}}, \mathcal{B}\right)$ preserves the symmetries.

Proof. This is a direct consequence of the above definitions, Theorem 5.7 and Proposition 2.18.

THEOREM 5.10. $\left[Q^{\star}\left(N_{\alpha}, L_{\alpha} ; \Sigma_{N_{\alpha}}, \mathcal{B}\right)\right]=\left[Q^{\star}\left(N_{\beta}, L_{\beta} ; \Sigma_{N_{\beta}}, \mathcal{B}\right)\right]$.

Proof. Applying Lemma 5.9 and Theorem 5.8 we see that, for $T=T_{\beta \alpha}$,

$$
f_{\beta \alpha}^{T}: Q^{\star}\left(N_{\alpha}, L_{\alpha} ; \Sigma_{N_{\alpha}}, \mathcal{B}\right) \rightarrow Q^{\star}\left(N_{\beta}, L_{\beta} ; \Sigma_{N_{\beta}}, \mathcal{B}\right)
$$

is a $\Sigma$-homotopy equivalence with $\Sigma$-homotopy inverse

$$
f_{\alpha \beta}^{T}: Q^{\star}\left(N_{\beta}, L_{\beta} ; \Sigma_{N_{\beta}}, \mathcal{B}\right) \rightarrow Q^{\star}\left(N_{\alpha}, L_{\alpha} ; \Sigma_{N_{\alpha}}, \mathcal{B}\right) .
$$

Indeed,

$$
\begin{aligned}
& H_{\alpha}: N_{\alpha} / L_{\alpha} \times[0,1] \rightarrow N_{\alpha} / L_{\alpha}, H_{\alpha}(x, \tau)=F_{\alpha}(x, 2 \tau T), \\
& H_{\beta}: N_{\beta} / L_{\beta} \times[0,1] \rightarrow N_{\beta} / L_{\beta}, H_{\beta}(x, \tau)=F_{\beta}(x, 2 \tau T),
\end{aligned}
$$

yield $f_{\alpha \beta}^{T} \circ f_{\beta \alpha}^{T} \stackrel{\Sigma}{\sim} i d_{N_{\alpha} / L_{\alpha}}$ and $f_{\beta \alpha}^{T} \circ f_{\alpha \beta}^{T} \stackrel{\Sigma}{\sim} i d_{N_{\beta} / L_{\beta}}$ respectively.

LEMmA 5.11. If $\left(N_{\alpha}, L_{\alpha}\right)$ and $\left(N_{\beta}, L_{\beta}\right)$ are $\Sigma$-invariant index pairs then $C_{\beta \alpha}^{t}$ is a $\Sigma$-invariant subset of $X$ for $t \geqslant 0$.

Proof. This is straightforward from Remark 2.12 and Proposition 2.2, as $C_{\beta \alpha}^{t}$ is an intersection of preimages of $\Sigma$-invariant subsets of $X$.

LEMmA 5.12. If $\left(N_{\alpha}, L_{\alpha}\right)$ and $\left(N_{\beta}, L_{\beta}\right)$ are $\Sigma$-invariant index pairs then, for $t>T_{\beta \alpha}$, the map $f_{\beta \alpha}^{t}: Q\left(N_{\alpha}, L_{\alpha} ; \Sigma_{N_{\alpha}}, \mathcal{B}\right) \rightarrow Q\left(N_{\beta}, L_{\beta} ; \Sigma_{N_{\beta}}, \mathcal{B}\right)$ preserves the symmetries.

Proof. This is a direct consequence of the above definition, Lemma 5.11, Theorem 5.7 and Proposition 2.19.

TheOREM 5.13. If $\left(N_{\alpha}, L_{\alpha}\right)$ and $\left(N_{\beta}, L_{\beta}\right)$ are $\Sigma$-invariant index pairs then

$$
\left[Q\left(N_{\alpha}, L_{\alpha} ; \Sigma_{N_{\alpha}}, \mathcal{B}\right)\right]=\left[Q\left(N_{\beta}, L_{\beta} ; \Sigma_{N_{\beta}}, \mathcal{B}\right)\right]
$$


Proof. Applying Lemma 5.12 and Theorem 5.8 we see that, for $T=T_{\beta \alpha}$,

$$
f_{\beta \alpha}^{T}: Q\left(N_{\alpha}, L_{\alpha} ; \Sigma_{N_{\alpha}}, \mathcal{B}\right) \rightarrow Q\left(N_{\beta}, L_{\beta} ; \Sigma_{N_{\beta}}, \mathcal{B}\right)
$$

is a $\Sigma$-homotopy equivalence with $\Sigma$-homotopy inverse

$$
f_{\alpha \beta}^{T}: Q\left(N_{\beta}, L_{\beta} ; \Sigma_{N_{\beta}}, \mathcal{B}\right) \rightarrow Q\left(N_{\alpha}, L_{\alpha} ; \Sigma_{N_{\alpha}}, \mathcal{B}\right)
$$

Indeed,

$$
\begin{aligned}
& H_{\alpha}: N_{\alpha} / L_{\alpha} \times[0,1] \rightarrow N_{\alpha} / L_{\alpha}, H_{\alpha}(x, \tau)=F_{\alpha}(x, 2 \tau T), \\
& H_{\beta}: N_{\beta} / L_{\beta} \times[0,1] \rightarrow N_{\beta} / L_{\beta}, H_{\beta}(x, \tau)=F_{\beta}(x, 2 \tau T),
\end{aligned}
$$

yield $f_{\alpha \beta}^{T} \circ f_{\beta \alpha}^{T} \stackrel{\Sigma}{\sim} i d_{N_{\alpha} / L_{\alpha}}$ and $f_{\beta \alpha}^{T} \circ f_{\alpha \beta}^{T} \stackrel{\Sigma}{\sim} i d_{N_{\beta} / L_{\beta}}$ respectively.

5.3. Properties of the homotopy Conley indexes with symmetries. In this section we formulate the properties of the above defined homotopy Conley indexes.

5.3.1. Normalization. Define the space with symmetries

$$
0=\left(\{\emptyset\}, \emptyset ; \Sigma_{\emptyset}, \mathcal{B}\right)
$$

where $\Sigma_{\emptyset}=\{\{\emptyset\}\} \times \mathcal{B}$. This space plays the role of the trivial space in the category of spaces with base point and symmetries over $\mathcal{B}$.

Proposition 5.14. The set $\emptyset \in I I S_{\Sigma}(\varphi)$ is a $\Sigma$-admissible isolated invariant set of the flow $\varphi$ and $\Sigma h(\emptyset, \varphi)=\Sigma^{\star} h(\emptyset)=[0]$.

Proof. First we observe that $(\emptyset, \emptyset)$ is a $\Sigma$-invariant index pair of the set $\emptyset$. Hence, according to our construction, we obtain $Q(\emptyset, \emptyset)=0=0^{\star}$.

5.3.2. Additivity. Consider isolated invariant $(\Sigma, \varphi)$-disjoint sets $S_{1}, S_{2} \in I I S(\varphi)$ of the flow $\varphi$.

Proposition 5.15. $\quad \Sigma^{\star} h\left(S_{1} \cup S_{2}, \varphi\right)=\Sigma^{\star} h\left(S_{1}, \varphi\right) \vee \Sigma^{\star} h\left(S_{2}, \varphi\right)$.

Proof. According to our assumptions there are index pairs $\left(N_{1}, L_{1}\right)$ and $\left(N_{2}, L_{2}\right)$ of $S_{1}$ and $S_{2}$ respectively such that $N_{1}$ and $N_{2}$ are $\Sigma$-disjoint, thus disjoint. It is known that $\left(N_{1} \cup N_{2}, L_{1} \cup L_{2}\right)$ is an index pair of $S_{1} \cup S_{2}$. Hence, due to Propositions 2.16 and 2.17 , we obtain

$$
\begin{aligned}
\Sigma^{\star} h\left(S_{1} \cup S_{2}, \varphi\right) & =\left[Q^{\star}\left(N_{1} \cup N_{2}, L_{1} \cup L_{2} ; \Sigma_{N_{1} \cup N_{2}}, \mathcal{B}\right)\right] \\
& =\left[\left(Q\left(N_{1}, L_{1} ; \Sigma_{N_{1}}, \mathcal{B}\right) \vee Q\left(N_{2}, L_{2} ; \Sigma_{N_{2}}, \mathcal{B}\right)\right)^{\star}\right] \\
& =\left[Q^{\star}\left(N_{1}, L_{1} ; \Sigma_{N_{1}}, \mathcal{B}\right) \vee Q^{\star}\left(N_{2}, L_{2} ; \Sigma_{N_{2}}, \mathcal{B}\right)\right] \\
& =\left[Q^{\star}\left(N_{1}, L_{1} ; \Sigma_{N_{1}}, \mathcal{B}\right)\right] \vee\left[Q^{\star}\left(N_{2}, L_{2} ; \Sigma_{N_{2}}, \mathcal{B}\right)\right] \\
& =\Sigma^{\star} h\left(S_{1}, \varphi\right) \vee \Sigma^{\star} h\left(S_{2}, \varphi\right) .
\end{aligned}
$$

Proposition 5.16. If $S_{1}, S_{2} \in I I S_{\Sigma}(\varphi)$ then $S_{1} \cup S_{2} \in I I S_{\Sigma}(\varphi)$ and

$$
\Sigma h\left(S_{1} \cup S_{2}, \varphi\right)=\Sigma h\left(S_{1}, \varphi\right) \vee \Sigma h\left(S_{2}, \varphi\right) .
$$

Proof. Our assumptions guarantee that there are $\Sigma$-invariant index pairs $\left(N_{1}, L_{1}\right)$ and $\left(N_{2}, L_{2}\right)$ of $S_{1}$ and $S_{2}$ respectively such that $N_{1}$ and $N_{2}$ are $\Sigma$-disjoint, thus disjoint. It is known that $\left(N_{1} \cup N_{2}, L_{1} \cup L_{2}\right)$ is an index pair of $S_{1} \cup S_{2}$, moreover these sets are $\Sigma$-invariant, as stated in Proposition 2.2, thus $S_{1} \cup S_{2} \in I I S_{\Sigma}(\varphi)$. 
Hence, due to Proposition 2.16, we have

$$
\begin{aligned}
\Sigma h\left(S_{1} \cup S_{2}, \varphi\right) & =\left[Q\left(N_{1} \cup N_{2}, L_{1} \cup L_{2} ; \Sigma_{N_{1} \cup N_{2}}, \mathcal{B}\right)\right] \\
& =\left[Q\left(N_{1}, L_{1} ; \Sigma_{N_{1}}, \mathcal{B}\right) \vee Q\left(N_{2}, L_{2} ; \Sigma_{N_{2}}, \mathcal{B}\right)\right] \\
& =\left[Q\left(N_{1}, L_{1} ; \Sigma_{N_{1}}, \mathcal{B}\right)\right] \vee\left[\left(N_{2}, L_{2} ; \Sigma_{N_{2}}, \mathcal{B}\right)\right] \\
& =\Sigma h\left(S_{1}, \varphi\right) \vee \Sigma h\left(S_{2}, \varphi\right) .
\end{aligned}
$$

5.3.3. Multiplicity. Consider spaces with symmetries $\left(X ; \Sigma_{X}, \mathcal{B}_{X}\right),\left(Y ; \Sigma_{Y}, \mathcal{B}_{Y}\right)$ and flows $\varphi_{X}: X \times \mathbf{R} \rightarrow X, \varphi_{Y}: Y \times \mathbf{R} \rightarrow Y$ which preserve the given symmetries.

We define the flow

$$
\varphi: X \times Y \times \mathbf{R} \rightarrow X \times Y, \varphi(x, y, t) \stackrel{\text { def }}{=}\left(\varphi_{X}(x, t), \varphi_{Y}(y, t)\right)
$$

which preserves the symmetries of the space $\left(X ; \Sigma_{X}, \mathcal{B}_{X}\right) \times\left(Y ; \Sigma_{Y}, \mathcal{B}_{Y}\right)$.

Moreover let $S_{X} \in I I S\left(\varphi_{X}\right)$ and $S_{Y} \in I I S\left(\varphi_{Y}\right)$. Then $S_{X} \times S_{Y} \in I I S(\varphi)$.

Proposition 5.17. $\quad \Sigma^{\star} h\left(S_{1} \times S_{2}, \varphi\right)=\Sigma^{\star} h\left(S_{X}, \varphi_{X}\right) \wedge \Sigma^{\star} h\left(S_{Y}, \varphi_{Y}\right)$.

Proof. Let $\left(N_{X}, L_{X}\right)$ and $\left(N_{Y}, L_{Y}\right)$ be index pairs of $S_{X}$ and $S_{Y}$ respectively. It is known that $\left(N_{X} \times N_{Y}, N_{X} \times L_{Y} \cup L_{X} \times N_{Y}\right)$ is an index pair of $S_{X} \times S_{Y}$. Hence, due to Proposition 2.17, we obtain

$$
\begin{aligned}
\Sigma^{\star} h\left(S_{1} \times S_{2}, \varphi\right) & =\left[Q^{\star}\left(N_{1} \times N_{2}, N_{1} \times L_{2} \cup L_{1} \times N_{2} ; \Sigma_{N_{1} \times N_{2}}, \mathcal{B}_{X} \times \mathcal{B}_{Y}\right)\right] \\
& =\left[\left(Q\left(N_{1}, L_{1} ; \Sigma_{N_{1}}, \mathcal{B}_{X}\right) \wedge Q\left(N_{2}, L_{2} ; \Sigma_{N_{2}}, \mathcal{B}_{Y}\right)\right)^{\star}\right] \\
& =\left[Q^{\star}\left(N_{1}, L_{1} \Sigma_{N_{1}}, \mathcal{B}_{X}\right) \wedge Q^{\star}\left(N_{2}, L_{2} ; \Sigma_{N_{2}}, \mathcal{B}_{Y}\right)\right] \\
& =\left[Q^{\star}\left(N_{1}, L_{1} ; \Sigma_{N_{1}}, \mathcal{B}_{X}\right) \wedge\left[Q^{\star}\left(N_{2}, L_{2} ; \Sigma_{N_{2}}, \mathcal{B}_{Y}\right)\right]\right. \\
& =\Sigma^{\star} h\left(S_{1}, \varphi\right) \wedge \Sigma^{\star} h\left(S_{2}, \varphi\right) .
\end{aligned}
$$

Proposition 5.18. If $S_{X} \in I I S_{\Sigma_{X}}\left(\varphi_{X}\right)$ and $S_{Y} \in I I S_{\Sigma_{Y}}(\varphi)$ then $S_{1} \times S_{2} \in$ $\operatorname{II} S_{\Sigma_{X \times Y}}(\varphi)$ and

$$
\Sigma h\left(S_{1} \times S_{2}, \varphi\right)=\Sigma h\left(S_{X}, \varphi_{X}\right) \wedge \Sigma h\left(S_{Y}, \varphi_{Y}\right) .
$$

PRoof. According to our assumptions there are $\Sigma$-invariant index pairs $\left(N_{X}, L_{X}\right)$ and $\left(N_{Y}, L_{Y}\right)$ of $S_{X}$ and $S_{Y}$ respectively. It is known that

$$
\left(N_{X} \times N_{Y}, N_{X} \times L_{Y} \cup L_{X} \times N_{Y}\right)
$$

is an index pair of $S_{X} \times S_{Y}$. From Remark 2.5 and Proposition 2.2 we conclude that these sets are $\Sigma$-invariant, thus $S_{1} \times S_{2} \in I I S_{\Sigma_{X \times Y}}(\varphi)$.

Hence, we have

$$
\begin{aligned}
\Sigma h\left(S_{1} \times S_{2}, \varphi\right) & =\left[Q\left(N_{1} \times N_{2}, N_{1} \times L_{2} \cup L_{1} \times N_{2} ; \Sigma_{N_{1} \times N_{2}}, \mathcal{B}_{X} \times \mathcal{B}_{Y}\right)\right] \\
& =\left[Q\left(N_{1}, L_{1} \Sigma_{N_{1}}, \mathcal{B}_{X}\right) \wedge Q\left(N_{2}, L_{2} ; \Sigma_{N_{2}}, \mathcal{B}_{Y}\right)\right] \\
& =\left[Q\left(N_{1}, L_{1} ; \Sigma_{N_{1}}, \mathcal{B}_{X}\right)\right] \wedge\left[Q\left(N_{2}, L_{2} ; \Sigma_{N_{2}}, \mathcal{B}_{Y}\right)\right] \\
& =\Sigma h\left(S_{1}, \varphi\right) \wedge \Sigma h\left(S_{2}, \varphi\right) .
\end{aligned}
$$

5.3.4. Continuation. Let us introduce in the space $X \times[0,1]$ the symmetries

$$
\Sigma_{X \times[0,1]}=\{(|a| \times\{\lambda\},(a)):(|a|,(a)) \in \Sigma\}
$$

and consider a flow $\varphi: X \times[0,1] \times \mathbf{R} \rightarrow X \times[0,1]$ which preserves the symmetries. Then the flows

$$
\varphi_{\lambda}: X \times \mathbf{R} \rightarrow X, \varphi(x, \lambda, t)=\left(\varphi_{\lambda}(x, t), \lambda\right), \text { for } \lambda \in[0,1],
$$

preserve the symmetries of the space $(X ; \Sigma, \mathcal{B})$. 
Moreover let $S \in \operatorname{IIS}(\varphi)$ and

$$
S_{i} \stackrel{\text { def }}{=}\{x \in X:(x, i) \in S\}, \text { for } i=0,1 .
$$

It is known that $S_{0} \in I I S\left(\varphi_{0}\right)$ and $S_{1} \in I I S\left(\varphi_{1}\right)$ are isolated invariant sets of $\varphi_{0}$ and $\varphi_{1}$ respectively.

REMARK 5.19. Let $(N, L)$ be a $\Sigma$-invariant index pair of the set $S$ and let $\lambda \in[0,1]$. Then

$$
\left(N_{\lambda}, L_{\lambda}\right)=(\{x \in X:(x, \lambda) \in N\},\{x \in X:(x, \lambda) \in L\})
$$

is a $\Sigma$-invariant index pair for the flow $\varphi_{\lambda}$.

Proof. It is easy to notice that $\left(N_{\lambda}, L_{\lambda}\right)$ is an index pair for $\varphi_{\lambda}$, as $\varphi$ preserves the set $X \times\{\lambda\}$.

We are going to show that $N_{\lambda}, L_{\lambda}$ are $\Sigma$-invariant subsets of $X$. Let $a \in \Sigma_{X}$ be such that $|a| \cap N_{\lambda} \neq \emptyset$. Then $(|a| \times\{\lambda\},(a)) \in \Sigma_{X \times[0,1]}$ and $|a| \times\{\lambda\} \cap N \neq \emptyset$. Hence $|a| \times\{\lambda\} \subset N$, as $N$ is a $\Sigma$-invariant subset of $X \times[0,1]$, thus $|a| \subset N_{\lambda}$. Analogously we obtain that $L_{\lambda}$ is a $\Sigma$-invariant subset of $X$.

The continuation property of the Conley index with symmetries can be formulated in the following way.

Proposition 5.20. $\quad \Sigma^{\star} h\left(S_{0}, \varphi_{0}\right)=\Sigma^{\star} h\left(S_{1}, \varphi_{1}\right)$.

Proposition 5.21. If $S \in I I S_{\Sigma_{X \times[0,1]}}(\varphi)$ then $S_{0} \in I I S_{\Sigma_{X}}\left(\varphi_{0}\right), S_{1} \in I I S_{\Sigma_{X}}\left(\varphi_{1}\right)$ and

$$
\Sigma h\left(S_{0}, \varphi_{0}\right)=\Sigma h\left(S_{1}, \varphi_{1}\right)
$$

The proofs of these propositions can be based on the construction of a homotopy equivalence given by D. Salamon $[\mathrm{S}]$ in chapters 6.2 and 6.3. It is elementary, but technically difficult, to show that the maps defined there preserve the symmetries induced in the quotient spaces. We omit the details which, in fact, repeat the arguments used in section 5.2 where we proved the correctness of the definitions of Conley indexes with symmetries.

5.4. Existence of $\Sigma$-invariant index pairs. An important problem is to compare the class of invariant sets of a flow which are $\Sigma$-invariant subsets of the phase space with the class of those invariant sets of the flow which admit a $\Sigma$-invariant isolating neighbourhood or a $\Sigma$-invariant index pair.

In general, when we do not impose any regularity conditions on the symmetries $\Sigma$, we cannot expect that a set $S \in I I S(\varphi) \cap P_{\Sigma}(X)$ possesses a $\Sigma$-invariant isolating neighbourhood or a $\Sigma$-invariant index pair. This can be easily observed, for example, when we consider, compact isolated invariant set $S$ of a flow $\varphi$ which is not an open subset of $X$ and the symmetries $\Sigma=\{S, X \backslash S\} \times \mathcal{B}$.

However, if the symmetries $\Sigma$ are regular in the sense of Definition 2.8 all those conditions describe the same class of sets. This will be studied in detail in this section.

Consider a space $(X ; \Sigma, \mathcal{B})$ and assume that the symmetries $\Sigma$ are regular. Moreover let $A \subset X$ be a compact isolating neighbourhood for a flow $\varphi: X \times \mathbf{R} \rightarrow X$ which preserves the symmetries. We denote $S=i n v(A, \varphi)$. 
THEOREM 5.22. The following conditions are equivalent:

(1) $S$ is a $\Sigma$-invariant subset of $X$,

(2) there is a compact $\Sigma$-invariant isolating neighbourhood $B \subset A$ of $S$,

(3) there exists a $\Sigma$-invariant regular index pair $(N, L)$ of $S$ such that $N \subset A$.

Proof. (3) $\Rightarrow(1)$ We have

$$
S=\operatorname{inv}(\operatorname{cl}(N \backslash L)) \subset \operatorname{int}(N \backslash L) \subset N \backslash L .
$$

Hence

$$
S=\operatorname{inv}(N \backslash L)=\bigcap_{t \in \mathbf{R}} \varphi^{-t}(N \backslash L),
$$

thus $S$ is a $\Sigma$-invariant subset of $X$ as the intersection of a family of $\Sigma$-invariant sets.

To prove the remaining part of the theorem we will adopt techniques used in $G$ equivariant Conley index theory.

Let $Y=X / R_{\Sigma}$ denote the quotient space and let $\pi: X \rightarrow X / R_{\Sigma}$ stand for the canonical projection. We define the flow

$$
\psi: Y \times \mathbf{R} \rightarrow Y, \psi([x], t)=[\varphi(x, t)] .
$$

$(1) \Rightarrow(2)$ Let $D=X \backslash \operatorname{int}(A)$. Then $\pi(D) \cap \pi(S)=\emptyset$. As $Y$ is a locally compact metric space, there is a compact set $V \subset Y \backslash \pi(D)$ such that $\pi(S) \subset \operatorname{int}(V)$. Hence

$$
S=\pi^{-1}(\pi(S)) \subset \pi^{-1}(\operatorname{int}(V)) \subset \operatorname{int}\left(\pi^{-1}(V)\right) \subset \pi^{-1}(V) \subset \operatorname{int}(A) \subset A,
$$

thus we can take $B=\pi^{-1}(V)$.

$(2) \Rightarrow(3)$ In Lemma 5.23 it is proved that $\pi(B)$ is an isolating neighbourhood of the set $\pi(S)$ for the flow $\psi$. Theorem 5.1 guarantees that there is a regular index pair $(P, Q)$, $P \subset \pi(B)$, of the set $\pi(S)$. The lemmas below say that

$$
(N, L)=\left(\pi^{-1}(N), \pi^{-1}(L)\right), N \subset B \subset A,
$$

is a regular index pair of $S$.

We formulate the lemmas we used in the proof above. We always assume (2).

LEMMA 5.23. The set $\pi(B)$ is a compact isolating neighbourhood of $\pi(S)$ for the flow $\psi$.

Proof. We see that $\pi(B)$ is compact and

$$
\pi(S) \subset \pi(\operatorname{int}(B)) \subset \operatorname{int}(\pi(B)) \subset \pi(B)
$$

because $\pi$ is an open map. We prove that $\pi(S)=i n v(\pi(B), \psi)$.

Let $y \in \operatorname{inv}(\pi(B), \psi)$. Then $y=\pi(x)$ for some $x \in X$. Hence

$$
\Phi(\pi(x), t)=\pi(\varphi(x, t)) \in \pi(B)
$$

for any $t \in \mathbf{R}$, so $\varphi(x, t) \in \pi^{-1}(\pi(B))=B$. Thus $x \in S$ and $y \in \pi(S)$.

Conversely, let $y \in \pi(S)$. Then $y=\pi(x)$ for some $x \in S$. Hence, for any $t \in \mathbf{R}$, we have $\psi(y, t)=\pi(\varphi(x, t)) \in \pi(S) \subset \pi(B)$, thus $y \in \operatorname{inv}(\pi(B))$.

Lemma 5.24. Let $(N, L) \subset(\pi(B), \pi(B))$ be an index pair of the set $\pi(S)$ for the flow $\psi$. Then $\left(N^{*}, L^{*}\right)=\left(\pi^{-1}(N), \pi^{-1}(L)\right)$ is an index pair of the set $S$ for the flow $\varphi$. 
Proof. First we observe that the sets $L^{*} \subset N^{*} \subset B$ are compact and

$$
S=\pi^{-1}(\pi(S)) \subset \pi^{-1}(\operatorname{int}(N \backslash L)) \subset \operatorname{int}\left(N^{*} \backslash L^{*}\right),
$$

hence $\operatorname{cl}\left(\left(N^{*} \backslash L^{*}\right) \subset B\right.$ is an isolating neighbourhood of $S$.

Let $x \in N^{*}$ and let $\varphi(x, t) \notin N^{*}$ for some $t>0$. Then $\pi(x) \in N$ and $\pi(\varphi(x, t))=$ $\psi(\pi(x), t) \notin N$. As $(N, L)$ is an index pair for $\psi$, there is $\tau \in[0, t]$ such that $\psi(\pi(x), \tau) \in L$, so $\varphi(x, \tau) \in L^{*}$.

Let $x \in L^{*}$ and $t \in \mathbf{R}$ be such that $\varphi(x, \tau) \in N^{*}$ for $\tau \in[0, t]$. Hence we have $\pi(x) \in L$ and $\pi(\varphi(x, \tau))=\psi(\pi(x), \tau) \in N$ for $\tau \in[0, t]$. As $(N, L)$ is an index pair for $\psi$, we have $\psi(\pi(x), t) \in L$ for $\tau \in[0, t]$, thus $\varphi(x, t) \in L^{*}$ for $\tau \in[0, t]$.

Lemma 5.25. Let $(N, L)$ and $\left(N^{*}, L^{*}\right)$ be as in Lemma 5.4 and let $\tau: N \rightarrow[0,+\infty]$ and $\tau^{*}: N^{*} \rightarrow[0,+\infty]$ be the exit time maps of the index pairs $(N, L)$ and $\left(N^{*}, L^{*}\right)$ respectively. Then $\tau^{*}=\tau \circ \pi$.

Proof. We notice that $N^{*} \backslash L^{*}=\pi^{-1}(N \backslash L)$, so for $x \in X$,

$$
x \in N^{*} \backslash L^{*} \text { if and only if } \pi(x) \in N \backslash L .
$$

The rest follows directly from the definition of the exit time map and the flows $\varphi$ and $\psi$.

Corollary 5.26. Let $S_{1}, S_{2} \in I I S(\varphi)$ be disjoint and $\Sigma$-invariant sets. Then $S_{1}$ and $S_{2}$ are $(\Sigma, \varphi)$-disjoint.

Proof. Indeed, as $S_{1}$ and $S_{2}$ are disjoint, they admit disjoint isolating neighbourhoods $A_{1}$ and $A_{2}$ respectively. Hence, Theorem 5.22(3) completes the proof.

6. Examples of the use of the $\Sigma^{\star} h$ index. In this section we calculate Conley indexes with symmetries of some isolated invariant sets and analyse them in relation to the behaviour of the flow. In particular we discuss the problem of the connecting orbit and the problem of deformation of the flow.

In the class $\mathcal{F}$ of vector fields on $\mathbf{R}^{2}$ which induce flows as solutions of associated ODEs we consider the class $\mathcal{F}_{\Sigma} \subset \mathcal{F}$ satisfying additional conditions. We say that $F=$ $\left(F_{1}, F_{2}\right) \in \mathcal{F}_{\Sigma}$ if

(1) $F((0,1))=(0,0)$,

(2) $F_{2}((x,-1))=0$ for $x \in \mathbf{R}$.

We show how the above restrictions affect the behaviour of the flow and how it can be observed in the context of Conley index with symmetries.

Let $\mathcal{B}=\{1,2,3\} \subset \mathbf{N}$ be the basic set of the generalized symmetries. We denote

$$
E \stackrel{\text { def }}{=} \mathbf{R} \times\{-1\}, P \stackrel{\text { def }}{=}(0,1)
$$

and

$$
(X ; \Sigma, \mathcal{B}) \stackrel{\text { def }}{=}\left(X ;\{(E, 1),(\{P\}, 2)\} \cup\left\{(\{x\}, 3): x \in \mathbf{R}^{2} \backslash(E \cup\{P\})\right\}, \mathcal{B}\right) .
$$

We notice that, for any $F \in \mathcal{F}_{\Sigma}$, the flow $\varphi: X \times \mathbf{R} \rightarrow X$ induced by the vector field $F$ preserves the symmetries $\Sigma$. Indeed, we see that

- condition (1) guarantees $\varphi^{t}(P)=P$ for $t \in \mathbf{R}$.

- condition (2) guarantees $\varphi^{t}(E)=E$ for $t \in \mathbf{R}$. 
Thus, for $x \in \mathbf{R}^{2} \backslash(E \cup\{P\})$ and $t \in \mathbf{R}$, we have $\varphi^{t}(x) \notin E \cup\{P\}$, as $\varphi^{t}: X \rightarrow X$ is a homeomorphism.

Let

$$
f(x, y) \stackrel{\text { def }}{=} \begin{cases}x^{2}+(y-1)^{2}+2 & \text { if } y \geqslant 1 \\ x^{2}+\left(3 y-y^{3}\right) & \text { if } y \in(-1,1) \\ x^{2}-(y+1)^{2}-2 & \text { if } y \leqslant-1\end{cases}
$$

and

$$
F(x, y) \stackrel{\text { def }}{=} \nabla f(x, y)= \begin{cases}(2 x, 2(y-1)) & \text { if } y \geqslant 1 \\ \left(2 x, 3\left(1-y^{2}\right)\right) & \text { if } y \in(-1,1) \in \mathcal{F}_{\Sigma} . \\ (2 x,-2(y+1)) & \text { if } y \leqslant-1\end{cases}
$$

The phase portrait of the flow $\varphi$ induced by the vector field $F$ is shown below.

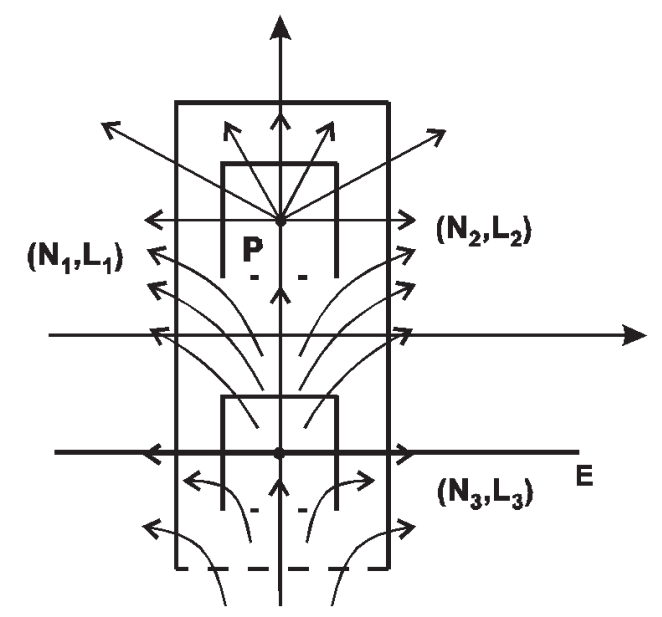

We notice that $\{(0,1)\} \in I I S_{\Sigma}(\varphi)$ and $A,\{(0,-1)\} \in I I S(\varphi) \backslash I I S_{\Sigma}(\varphi)$, where $A=$ $\{0\} \times[-1,1]$, are compact isolated invariant sets of the flow $\varphi$. Moreover

- $\left(N_{1}, L_{1}\right) \stackrel{\text { def }}{=}([-1,1] \times[-2,2],\{-1,1\} \times[-2,2] \cup[-1,1] \times\{2\})$ is an index pair for $A$,

- $\left(N_{2}, L_{2}\right) \stackrel{\text { def }}{=}([-1 / 2,1 / 2] \times[1 / 2,3 / 2],\{-1 / 2,1 / 2\} \times[1 / 2,3 / 2] \cup[-1 / 2,1 / 2] \times\{3 / 2\})$ is an index pair for $\{(0,1)\}$,

- $\left(N_{3}, L_{3}\right) \stackrel{\text { def }}{=}([-1 / 2,1 / 2] \times[-3 / 2,-1 / 2],\{-1 / 2,1 / 2\} \times[-3 / 2,-1 / 2] \cup[-1 / 2,1 / 2] \times$ $\{-1 / 2\})$ is an index pair for $\{(0,-1)\}$.

This allows us to calculate the Conley indexes with symmetries of these sets. Applying the notation of section 4 we obtain:

- $\Sigma^{\star} h(A, \varphi)=\left[\left(U, \star ; \Sigma_{U}, \mathcal{B}\right)\right]$,

- $\Sigma^{\star} h(\{(0,1)\}, \varphi)=\left[\left(Z, \star ; \Sigma_{Z}, \mathcal{B}\right)\right]$

- $\Sigma^{\star} h(\{(0,-1)\}, \varphi)=\left[\left(Y, \star ; \Sigma_{Y}, \mathcal{B}\right)\right]$.

We see that these sets have different Conley indexes with symmetries while their Conley indexes

are trivial.

$$
h(A, \varphi)=h(\{(0,1)\}, \varphi)=h(\{(0,-1)\}, \varphi)=[(\{\star\}, \star)],
$$


Furthermore, an elementary computation shows that according to Definition 5.4 the sets $N_{2}$ and $N_{3}$ are $\Sigma$-disjoint, so $\{(0,-1)\},\{(0,1)\} \in I I S(\varphi)$ are $(\Sigma, \varphi)$-disjoint.

Connecting orbits problem. Now, we study the problem of existence of additional stationary points or connecting orbits.

Let $g: \mathbf{R}^{2} \rightarrow \mathbf{R}$ be a function of class $C^{2}$ and $R>r>0$ such that

1. $g_{\mid B((0,-1), r)}=f_{\mid B((0,-1), r)}$ and $g_{\mid B((0,1), r)}=f_{\mid B((0,1), r)}$,

2. $g_{\mid \mathbf{R}^{2} \backslash B((0,0), R)}=f_{\mid \mathbf{R}^{2} \backslash B((0,0), R)}$.

Moreover let $\psi: \mathbf{R}^{2} \times \mathbf{R} \rightarrow \mathbf{R}^{2}$ denote the flow induced by $\nabla g: \mathbf{R}^{2} \rightarrow \mathbf{R}^{2}$.

THEOREM 6.1. Under the above assumptions, either

(1) there is $x \in B(0, R) \backslash\{(0,-1),(0,1)\}$ which is a stationary point of the flow $\psi$ or

(2) there is $x \in B(0, R) \backslash\{(0,-1),(0,1)\}$ such that

$$
\lim _{t \rightarrow-\infty} \psi^{t}(x)=(0,1) \text { and } \lim _{t \rightarrow+\infty} \psi^{t}(x)=(0,-1)
$$

which induces the orbit connecting the points $(0,1)$ and $(0,-1)$.

Proof. We set $B \stackrel{\text { def }}{=} B((0,0), 2 R)=\left\{x \in \mathbf{R}^{2}:|x| \leqslant 2 R\right\}$. By our observations, we have

$$
\begin{aligned}
\Sigma^{\star} h(\{(0,1)\}, \psi) & =\Sigma^{\star} h(\{(0,1)\}, \varphi), \\
\Sigma^{\star} h(\{(0,-1)\}, \psi) & =\Sigma^{\star} h(\{(0,1)\}, \varphi), \\
\Sigma^{\star} h(\operatorname{inv}(B), \psi) & =\Sigma^{\star} h(\operatorname{inv}(B), \varphi) .
\end{aligned}
$$

Hence by Lemma 4.3 we obtain

$$
\Sigma^{\star} h(\operatorname{inv}(B), \psi) \neq \Sigma^{\star} h(\{(0,1)\}, \psi) \vee \Sigma^{\star} h(\{(0,-1)\}, \psi) .
$$

We consider two cases:

1. $(\nabla g)^{-1}(0) \cap B \backslash\{(0,-1),(0,1)\} \neq \emptyset$. This is the $(1)$ of the theorem.

2. $(\nabla g)^{-1}(0) \cap B \backslash\{(0,-1),(0,1)\}=\emptyset$. Applying the additivity of the Conley index with symmetries we obtain

$$
\operatorname{inv}(B, \psi) \neq\{(0,-1),(0,1)\}
$$

hence there exists $x \in \operatorname{inv}(A, \psi) \backslash\{(0,-1),(0,1)\}$. As the flow is generated by a gradient vector field and $(0,-1),(0,1)$ are its only stationary points in $B$, it follows that $x$ induces the required connecting orbit.

Flow deformation problem. In the context of bifurcation phenomena a parametrized family of flows is studied. We show that the Conley index with symmetries can be used to verify whether one flow can be deformed to another one without disturbing a fixed isolating neighbourhood.

Set

$$
k(x, y) \stackrel{\text { def }}{=} f(x,-y)
$$

Then

$$
K(x, y)=\nabla k(x, y)=\left(F_{1}(x,-y),-F_{2}(x,-y)\right) \in \mathcal{F}_{\Sigma}
$$


hence

$$
K(x, y)= \begin{cases}(2 x, 2(1-y)) & \text { if } y \geqslant 1 \\ \left(2 x, 3\left(y^{2}-1\right)\right) & \text { if } y \in(-1,1) \\ (2 x, 2(1+y)) & \text { if } y \leqslant-1\end{cases}
$$

The phase portrait of the flow $\phi$ induced by the vector field $K$ is shown below.

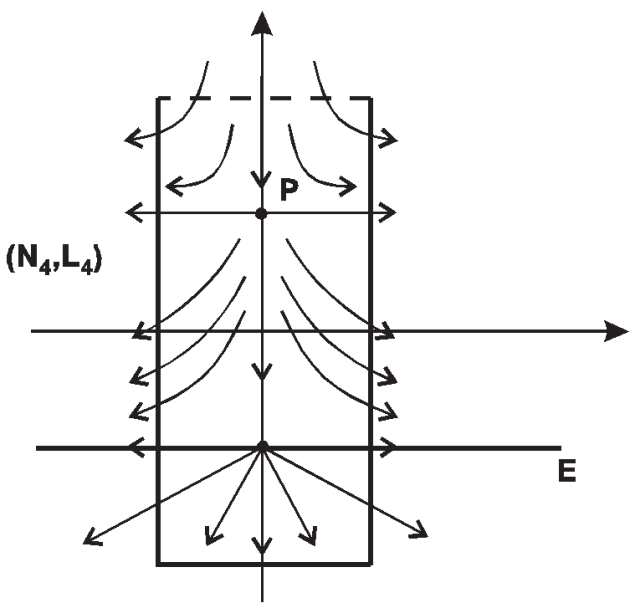

We see that the set

$$
A \stackrel{\text { def }}{=}\{0\} \times[-1,1] \in I I S(\phi) \backslash I I S_{\Sigma}(\phi)
$$

is a compact isolated invariant set of the flow $\phi$ and

$$
\left(N_{4}, L_{4}\right) \stackrel{\text { def }}{=}([-1,1] \times[-2,2],\{-1,1\} \times[-2,2] \cup[-1,1] \times\{-2\})
$$

is its index pair. Hence, applying the notation of section 4, we obtain that the Conley index of $A$ is

$$
\Sigma^{\star} h(A, \phi)=\left[\left(W, \star ; \Sigma_{W}, \mathcal{B}\right)\right]
$$

while its Conley index $h(A, \phi)=[(\{\star\}, \star)]$ is trivial.

Let $N \subset X$ be any isolating neighbourhood of $A$.

Proposition 6.2. There is no homotopy $H: \mathbf{R}^{2} \times[0,1] \rightarrow \mathbf{R}^{2}$ such that

1. $H_{0}=F$ and $H_{1}=K$,

2. $H_{s} \in \mathcal{F}_{\Sigma}$ for $s \in[0,1]$,

3. $N$ is an isolating neighbourhood for the flow $\varphi_{H_{s}}$ induced by the vector field $H_{s}$, for any $s \in[0,1]$.

Proof. This is a straightforward consequence of the continuation property of the Conley index with symmetries. Indeed $A=\operatorname{inv}(N, \varphi)=\operatorname{inv}(N, \phi)$ and $\Sigma^{\star} h(A, \varphi) \neq$ $\Sigma^{\star} h(A, \phi)$ as shown in Lemma 4.3 .

Proposition 6.3. There exists a homotopy $H: \mathbf{R}^{2} \times[0,1] \rightarrow \mathbf{R}^{2}$ such that

1. $H_{0}=F$ and $H_{1}=K$,

2. $H_{s} \in \mathcal{F}$ for $s \in[0,1]$, 
3. $N$ is an isolating neighbourhood for the flow $\varphi_{H_{s}}$ induced by the vector field $H_{s}$, for any $s \in[0,1]$.

Proof. We construct the required homotopy which connects $F$ and $K$ in such a way that $N$ is an isolating neighbourhood for the associated gradient flows.

This homotopy is obtained as the composition of a deformation $\left(H^{1}\right)$, a rotation $\left(H^{2}\right)$ and a deformation $\left(H^{3}\right)$ defined below.

Let $H^{1}: \mathbf{R}^{2} \times[0,1] \rightarrow \mathbf{R}$,

$$
H^{1}(x, y, s)= \begin{cases}x^{2}+(y-(1-s))^{2}+2(1-s)^{3} & \text { if } y \geqslant 1-s \\ x^{2}+\left(3(1-s)^{2} y-y^{3}\right) & \text { if } y \in(s-1,1-s) \\ x^{2}-(y+(1-s))^{2}-2(1-s)^{3} & \text { if } y \leqslant s-1\end{cases}
$$

and $H^{2}: \mathbf{R}^{2} \times[0,1] \rightarrow \mathbf{R}$,

$$
H^{2}(x, y, s)=H_{0}^{1}\left(O_{s}(x, y)\right)
$$

where

$$
O_{s}=\left(\begin{array}{cc}
\cos \pi s & -\sin \pi s \\
\sin \pi s & \cos \pi s
\end{array}\right)
$$

is the rotation through $s \cdot \pi$ and finally $H^{3}: \mathbf{R}^{2} \times[0,1] \rightarrow \mathbf{R}$,

$$
H^{3}(x, y, s)= \begin{cases}x^{2}-(y-s)^{2}-2 s^{3} & \text { if } y \geqslant s \\ x^{2}+\left(y^{3}-3 s^{2} y\right) & \text { if } y \in(-s, s) \\ x^{2}+(y+s)^{2}+2 s^{3} & \text { if } y \leqslant-s\end{cases}
$$

The stages of deformation of $\varphi$ to $\phi$ are presented below.
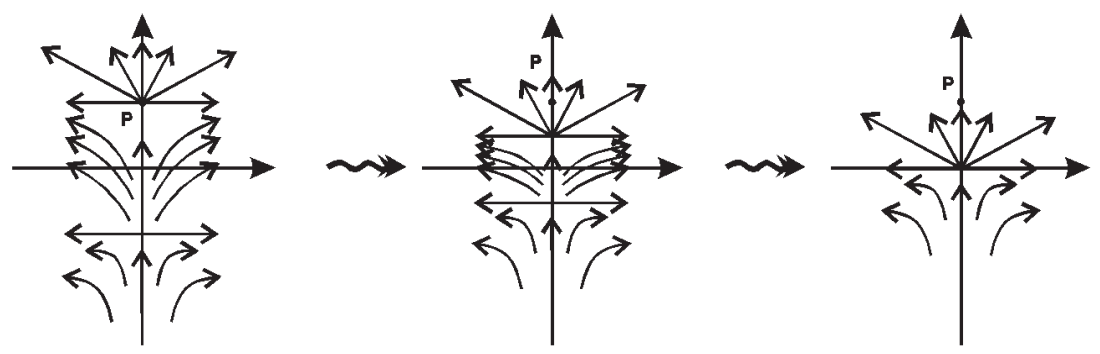

Phase portrait of the gradient flow along the deformation $H^{1}$
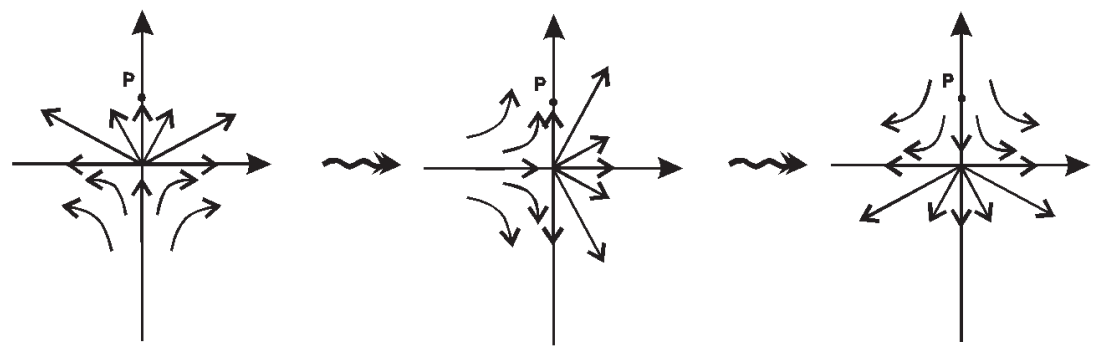

Phase portrait of the gradient flow along the rotation $H^{2}$ 


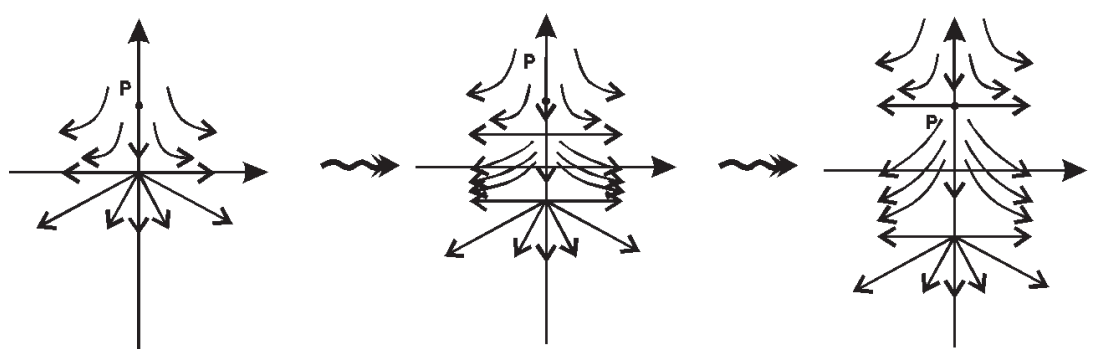

Phase portrait of the gradient flow along the deformation $H^{3}$

\section{References}

[B] T. BARTsCh, Topological Methods for Variational Problems with Symmetries, Springer, Berlin 1993.

[C] C. C. Conley, Isolated Invariant Sets and Morse Index, CBMS Regional Confer. Ser. in Math., Amer. Math. Soc., Providence, R.I., 1978.

[CZ] C. C. Conley and E. Zehnder, A Morse Type Index Theory for Flows and Periodic Solutions to Hamiltonian Systems, Comm. Pure Appl. Math. 37 (1984), 207-253.

[E] R. Engelking, General Topology, PWN, Warsaw 1977.

[F] A. Floer, A Refinement of the Conley Index and an Application to the Stability of Hyperbolic Invariant Sets, Ergod. Th. and Dynam. Syst. 7 (1988), 93-103.

[FZ] A. Floer and E. Zehnder, The Equivariant Conley Index and Bifurcation of Periodic Solution of Hamiltonian Systems, Ergod. Th. and Dynam. Syst. 8* (1988), 87-97.

[G] K. GȨBA, Degree for Gradient Equivariant Maps and Equivariant Conley Index, Topological Nonlinear Analysis II, Birkhäuser, Boston - Basel - Berlin 1997, 247-272.

[M] K. Mischaikow, Conley Index Theory, Lect. Notes in Math. 1609, 119-207, Springer, Berlin 1995.

[RS] J. Robbin and D. Salamon, Dynamical Systems, Shape Theory and the Conley Index, Ergod. Th. and Dynam. Syst. 8* (1988), 375-393.

[R] K. P. Rybakowski, The Homotopy Index and Partial Differential Equations, Springer, Berlin 1987.

[S] D. Salamon, Connected Simple Systems and the Conley Index of Isolated Invariant Sets, Trans. Amer. Math. Soc. 291 (1985), 1-41.

[Sz] A. Szymczak, The Conley Index for Discrete Semidynamical Systems, Top. Appl. 66 (1995), 215-240.

[W] T. WAŻEWski, Sur un principe topologique de l'examen de l'allure asymptotique des intégrales des équations différentielles, Ann. Soc. Polon. Math. 20 (1947), 249-313.

[Wh] G. W. WhiteheAd, Elements of Homotopy Theory, Springer, New York 1978. 